\title{
Performance of new BOC-AW-modulated signals for GNSS system
}

\author{
Mustapha Flissi ${ }^{*}$, Khaled Rouabah', Djamel Chikouche ${ }^{2}$, Abdehalim Mayouf ${ }^{3}$ and Salim Atia ${ }^{1}$
}

\begin{abstract}
In this paper, we propose efficient signal waveforms (WFs) with optimized spectrum for multipath (MP) mitigation and jamming reduction in global navigation satellite system. These WFs are based on the use of sine-phased binary offset carrier (BOC) with adjustable width (BOC-AW). They are generated by adjusting the width of the three-level $W F\{-1,0,1\}$. By exploiting the three-level BOC-AW subcarriers in sine-phasing signal model, we developed several forms of BOC-AW by means of superposition and width adjustment. The resulting power spectral densities and autocorrelation functions of the proposed WFs were calculated and introduced. Also calculated were the spectral separation coefficients (SSCs) and the Cramér-Rao lower bounds (CRLBs). The SSCs and CRLBs prove the efficiency of the proposed WFs in terms of interference separation. In addition, the simulation results show that the proposed WFs present better performances in MP mitigation compared to the WFs adopted by the Galileo and global positioning system modernization.
\end{abstract}

Keywords: GPS, Galileo, Multipath, BOC, MBOC, TMBOC, DLL, PN

\section{Introduction}

The binary offset carrier (BOC) modulation represents a serious candidate for global navigation satellite system (GNSS), especially for future global positioning system (GPS). Proposed by [1], the BOC modulation has several advantages compared to the traditional binary phase shift keying (BPSK), such as good spectral efficiency, high accuracy, enhanced multipath (MP) resolution [2], and better anti-jamming performances [3]. Other forms of modulation derived from the BOC concept are also used for new GPS and Galileo systems, such as quaternary phase shift keying modulation in L5 GPS signals [4], alternative BOC modulation in E5 Galileo signals [4], multiplexed BOC (MBOC) modulation with composite BOC (CBOC) implementation for Galileo, and timemultiplexed BOC (TMBOC) implementation for future GPS L1C [5]. Although these modulations are an important innovation for GNSS systems, there are other modulations of great interest, such as binary coded symbol (BCS) modulation [6,7], composite binary coded symbols (CBCS) modulation [7], quadrature multiplexed BOC modulation

\footnotetext{
*Correspondence: flissi_mus@yahoo.fr

'LMSE Laboratory, Electronics Department, University of Bordj Bou Arréridj, Bordj Bou, Arréridj, Algeria

Full list of author information is available at the end of the article
}

[8], minimum shift keying BOC modulation [9], multilevel subcarrier modulation specifically for three-level or tertiary offset carrier (TOC) subcarrier modulation and five-level signals or 8-PSK subcarrier modulation [9,10], and m-PSK BOC modulation [11]. The 8-PSK signals achieve mostly better performances at lower bandwidth than comparable signal types. The effort to search for new signal waveforms (WFs) for navigation continues in order to propose a WF that has lower levels of interference with existing signals with an insurance of better performances in terms of MP mitigation and jamming reduction. In this paper, we propose efficient WFs for GNSS system which are labeled as sine-phased binary offset carrier with adjustable width (BOC-AW). These WFs are three-level $(-1,0,1)$ and based on the sine-phased BOC concept with adjustable pulse width within each subcarrier half cycle. A judicious choice of the pulse widths of the BOC-AW general mathematical model provides the general types of BOC and TOC WFs. The purpose of the proposed WFs is to eliminate components of the side lobes near the main lobe and at the same time increase the other side lobes of higher frequencies (far away from the main lobe) in order to get better performance in terms of MP and interference mitigation. 
BOC-AW $\left(p, q, \alpha^{(M)}\right)$ modulation presents a sharper main peak due to the larger number of transitions of the signal in the chip interval, which obviously corresponds to a greater slope of the discrimination function, allowing a reduction of MP effect. Also, BOC-AW modulations show better performances than $\operatorname{BOC}(p, p)$ and $\operatorname{BOC}(p, 1)$ modulations for $p>>1$ with regard to the receiving band. Moreover, both latter modulations have inconveniences. In fact, $\operatorname{BOC}(p, q)$ WFs $(q>>1)$ need the use of several generator polynomials in contrast to the case where $q=1$, which uses only two generator polynomials. Also, it has been found that the tracking loop design for $\operatorname{BOC}(p, 1)$ with $p>>1$ may be more problematic than for $\operatorname{BOC}(1,1)$, especially with the conventional delay locked loop (DLL) algorithm with a narrow correlator. In effect, the $\operatorname{BOC}(p, 1) \mathrm{ACFs}$ with $p>>1$, in contrast to that of $\operatorname{BOC}(1,1)$ modulation, produce several side peaks which complicate the DLL locking operation. The proposed WFs present also a better spectral separation and MP mitigation. Furthermore, they have better resistance against noise and jammer, and they can be used in conjunction with other MP mitigation techniques [12-15] for performance improvement.

This paper is organized as follows: firstly, we present the concept and the properties of BOC-AW-modulated WFs. Secondly, a general expression of the theoretical $\mathrm{ACF}$ and power spectral density (PSD) of BOC-AW WFs are presented. We present also the influence of pulse width adjustment on the structure of BOC-AW WF PSDs in comparison with their influence on that of BOC WF PSDs. Finally, the main performances of these proposed WFs are discussed and compared with the existing $\mathrm{BOC}$ and $\mathrm{MBOC}$ ones.

\section{Proposed WFS and their properties}

$\mathrm{BOC}$ is a square WF subcarrier modulation, where a signal $s(t)$ (the signal which is going to be modulated) is multiplied by a square WF subcarrier of frequency $f_{s}$. Formally, the BOC-modulated signal $s_{\mathrm{BOC}}(t)$ can be written as the product of $s(t)$ and $\operatorname{sign}\left(\sin \left(2 \pi f_{s} t\right)\right)[1,2]$.

For GNSS signals, the notation $\operatorname{BOC}(p, q)$ is used, where $p$ and $q$ are two indices satisfying the relationships

$$
p=f_{s}[\mathrm{MHz}] / 1.023[\mathrm{MHz}]
$$

and

$$
q=f_{c}[\mathrm{MHz}] / 1.023[\mathrm{MHz}],
$$

where $f_{c}$ is the chip rate of $s(t)$.

The proposed BOC-AW subcarriers are three-level $(-1,0,1)$ WFs with greater number of pulses in each subcarrier half cycle compared to the $\operatorname{BOC}(1,1)$, TOC, and $\mathrm{MBOC}$ ones. The spreading signal in BOC-AW can be expressed as follows:

$$
s(t)=\sum_{k=-\infty}^{+\infty} c_{k} \times s_{\mathrm{BOC}-\mathrm{AW}}\left(p, q, \alpha^{(M)}\right)\left(t-k n T_{s}\right)
$$

for $n$ (equal $2 p / q$ ) even, and

$$
s(t)=\sum_{k=-\infty}^{+\infty}(-1)^{k} c_{k} \times s_{\mathrm{BOC}-\mathrm{AW}}\left(p, q, \alpha^{(M)}\right)\left(t-k n T_{s}\right)
$$

for $n$ odd, where $T_{s}$ is the half period of the subcarrier, $n$ is the number of half period $T_{s}$ during one code chip period $T_{c}, C_{k}$ is the $k^{i-t h}$ chip of the PRN code with frequency $f_{\mathrm{c}}, s_{\mathrm{BOC}-\mathrm{AW}(p, q, \alpha}^{(M)}(t)$ is the proposed subcarrier WF with parameters $f_{s}, f_{c}$, and $\alpha^{(M)}$ with $\alpha^{(M)}=\left[\alpha_{1}, \alpha_{2}, \ldots, \alpha_{M}\right]$, $0 \leq \alpha_{1}<\alpha_{2}<\ldots<\alpha_{\mathrm{M}} \leq 1$, and $M=\{2,4,6\}$. It can be given as

$$
s_{\mathrm{BOC}-\mathrm{AW}\left(p, q, \alpha^{(M)}\right)}(t)=\sum_{m=0}^{n-1} \sum_{l=1}^{M / 2} \sum_{i=0}^{1}(-1)^{m} p_{l, i}\left(t-m T_{s}\right) .
$$

$P_{l, i}(t)$ is a square WF given as

$$
P_{l, i}(t)= \begin{cases}\left(2 i+(-1)^{i} \alpha_{(2 l+i-1)}\right) \\ 1 \quad \times \frac{T_{s}}{2} \leq t<\left(2 i+(-1)^{i} \alpha_{(2 l-i)}\right) \times \frac{T_{s}}{2} \\ 0 \quad \text { otherwise. }\end{cases}
$$

The forms of $\left.s_{\mathrm{BOC}-\mathrm{AW}(\mathrm{p}, \mathrm{q}, \alpha}{ }^{(M)}\right)(t)$ derived from the general model in Equation 3 for the different values of $M$ and with judicious choice of $\alpha^{(M)}$ are illustrated in Figure 1.

The expression of $\operatorname{BOC}(p, q)$ subcarrier used in Galileo and modernized GPS can be obtained easily from Equation 3 (corresponding to our proposed WFs) with $M=2$ and $\alpha^{(2)}=\left[\alpha_{1}=0, \alpha_{2}=1\right]$ (see Figure 1a). Similarly, the expression of $\operatorname{TOC}(p, q, \alpha)[9,10]$ can also be determined from the same equation with $M=2$ and $\alpha^{(2)}=\left[\alpha_{1}=\alpha, \alpha_{2}=1\right]$ (see Figure 1b).

As mentioned before, the BOC-AW WFs depend on several parameters. In order to get the optimization of the GNSS receiver performances in terms of noise, MP, and jamming reduction, a judicious choice of those parameters is needed. The most advantageous WFs are selected according to the PSD distribution over the frequency range, as we are going to see in the next section. 


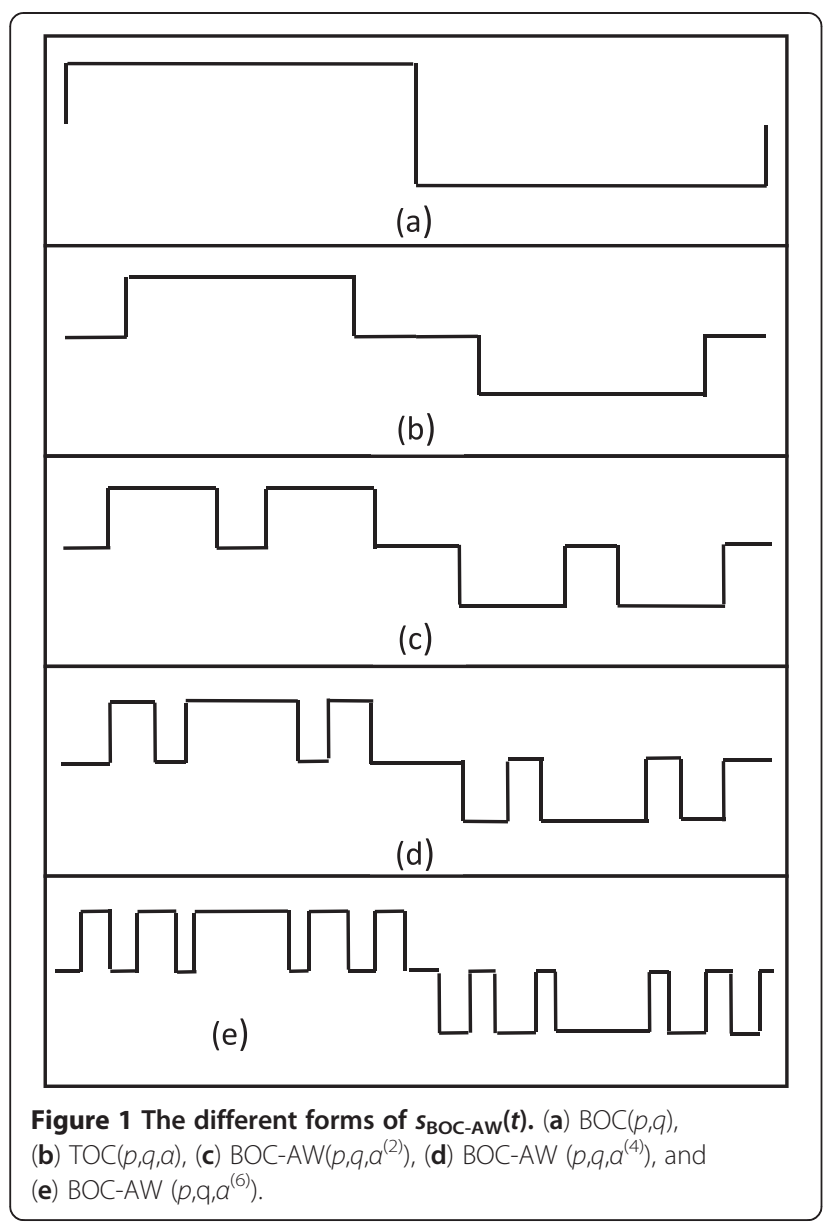

An example of three WFs is illustrated in Figure 1 (see Figure 1c,d,e). The WF of BOC-AW $\left(p, q, \alpha^{(4)}\right)$ (Figure 1d) can be determined by the superposition of two WFs of $\operatorname{BOC}-\mathrm{AW}\left(p, q, \alpha^{(2)}\right)$ (Figure 1c), one being defined by the factors $\alpha_{1}$ and $\alpha_{2}$ and the other by $\alpha_{3}$ and $\alpha_{4}=1$. Finally, $\operatorname{BOC}-\mathrm{AW}\left(p, q, \alpha^{(6)}\right)$ WF (Figure 1e) can be regarded as the superposition of the three WFs of BOC-AW $\left(p, q, \alpha^{(2)}\right)$ defined respectively by the pairs $\left(\alpha_{1}, \alpha_{2}\right),\left(\alpha_{3}, \alpha_{4}\right)$, and $\left(\alpha_{5}, \alpha_{6}=1\right)$.

\section{The BOC-AW WFS PSDs}

Under the assumption that all the symbols are statistically independent and equally probable, the PSDs of BOC-AW WFs with anti-polar binary code sequence can be established as follows:

$$
G_{\left(p, q, \alpha^{(M)}\right)}(f)=\frac{\left|S_{\mathrm{BOC}-\mathrm{AW}\left(p, q, \alpha^{(M)}\right)}(f)\right|^{2}}{T_{c}}
$$

where $S_{\mathrm{BOC}-\mathrm{AW}(p, q, \alpha}^{(\mathrm{M})}(f)$ is the Fourier transform of $s_{\mathrm{BOC}-\mathrm{AW}(p, q, \alpha}^{(M)}(t)$. The 'Appendix' shows the computation details of the PSDs of BOC-AW WFs which are given as follows:

$$
G_{\left(p, q, \alpha^{(M)}\right)}(f)=\frac{\left\{\sum_{l=1}^{M}(-1)^{l+1} \sin \left[\frac{\pi f}{2 f_{\mathrm{s}}}\left(1-\alpha_{\mathrm{l}}\right)\right]\right\}^{2} \sin ^{2}\left(\frac{\pi f}{f_{c}}\right)}{\mathrm{T}_{\mathrm{c}}\left(\alpha^{\prime}\right)(\pi \mathrm{f})^{2} \cos ^{2}\left(\frac{\pi f}{2 \mathrm{f}_{\mathrm{s}}}\right)}
$$

for $n$ even and

$$
G_{\left(p, q, \alpha^{(M)}\right)}(f)=\frac{\left\{\sum_{l=1}^{M}(-1)^{1+1} \sin \left[\frac{\pi f}{2 f_{s}}\left(1-\alpha_{l}\right)\right]\right\}^{2} \cos ^{2}\left(\frac{\pi f}{f_{c}}\right)}{T_{c}\left(\alpha^{\prime}\right)(\pi f)^{2} \cos ^{2}\left(\frac{\pi f}{2 f_{s}}\right)}
$$

for $n$ odd.

In Equations 6 and 7, $\alpha^{\prime}$ indicates the active time where the signal adopts the values -1 and 1 , and it is given as

$$
\alpha^{\prime}=\sum_{\mathrm{m}=1}^{\mathrm{M}}(-1)^{\mathrm{m}} \alpha_{\mathrm{m}}
$$

where $0<\alpha^{\prime} \leq 1$.

For $M=2$ and $\alpha^{(2)}=\left[\alpha_{1}=0, \alpha_{2}=1\right]$, Equations 6 and 7 become

$$
G_{\left(p, q, \alpha^{(2)}\right)}(f)=\frac{\left\{\sin \left[\frac{\pi f}{2 f_{s}}\right]\right\}^{2} \sin ^{2}\left(\frac{\pi f}{f_{c}}\right)}{T_{c}(\pi f)^{2} \cos ^{2}\left(\frac{\pi f}{2 f_{s}}\right)}
$$

for $n$ even and

$$
G_{\left(p, q, \alpha^{(2)}\right)}(f)=\frac{\left\{\sin \left[\frac{\pi f}{2 f_{s}}\right]\right\}^{2} \cos ^{2}\left(\frac{\pi f}{f_{c}}\right)}{T_{c}(\pi f)^{2} \cos ^{2}\left(\frac{\pi f}{2 f_{s}}\right)}
$$

for $n$ odd.

The resultant expressions (9) and (10) correspond to those of $\operatorname{BOC}(p, q)$ PSDs. Similarly, the PSD of $\operatorname{TOC}(p, q, \alpha)$ is also obtained from Equations 6 and 7, with $M=2$, $\alpha^{(2)}=\left[\alpha_{1}=\alpha, \alpha_{2}=1\right]$, and $\alpha^{\prime}=1-\alpha$. It is given as $[7,8]$ as follows:

$$
G_{(p, q, \alpha)}(f)=\frac{\left\{\sin \left[\frac{\pi f}{2 f_{s}}(1-\alpha)\right]\right\}^{2} \sin ^{2}\left(\frac{\pi f}{f_{c}}\right)}{T_{c}\left(\alpha^{\prime}\right)(\pi f)^{2} \cos ^{2}\left(\frac{\pi f}{2 f_{s}}\right)}
$$


for $n$ even and

$$
G_{(p, q, \alpha)}(f)=\frac{\left\{\sin \left[\frac{\pi f}{2 f_{s}}(1-\alpha)\right]\right\}^{2} \cos ^{2}\left(\frac{\pi f}{f_{c}}\right)}{T_{c}\left(\alpha^{\prime}\right)(\pi f)^{2} \cos ^{2}\left(\frac{\pi f}{2 f_{s}}\right)}
$$

for $n$ odd.

The sine-phased $\operatorname{BOC}(p, p)$ signal is characterized by $f_{s}=f_{c}=p \times 1.023 \mathrm{MHz}$ and $n=2$. For $n$ even, the difference between the PSD of BOC-AW in Equation 6 and the $\operatorname{PSD}$ of $\operatorname{BOC}(p, p)$ in Equation 9 lies in the sine functions that contain the $\alpha^{(M)}$ factors.

The aim is to use these functions to remove, by a judicious choice of the $\alpha^{(M)}$ factors, some components of frequencies $f=k f_{s}$ in the $\operatorname{PSD}$ of $\operatorname{BOC}(p, p)$, more precisely those corresponding to the maxima of secondary lobes $\left(3 f_{s}, 5 f_{s}, 9 f_{s} \ldots\right)$ that are nearer the principal lobes. Thus, we force the reduced power to be translated towards higher frequencies, which causes a positive impact on GNSS receiver performances.

To remove the frequency components $k f_{s}$ in the PSD of BOC-AW WFs, we solve the following equations:

$$
\left\{\sum_{l=1}^{M}(-1)^{1+1} \sin \left[\frac{\pi k f_{s}}{2 f_{s}}\left(1-\alpha_{l}\right)\right]\right\}^{2}=0
$$

which for $k$ odd can be further simplified to

$$
\left\{\sum_{l=1}^{M}(-1)^{1+1} \cos \left(\frac{\pi k}{2} \alpha_{l}\right)\right\}^{2}=0 .
$$

Depending on the values of $M$ and $\alpha^{(M)}$, four cases can be considered.
Case 1: $M=2,0<a_{1}<1$, and $a_{2}=1$

For this case, we find the TOC signal (Figure 1b) and a system of equations given by

$$
\left\{\begin{array}{l}
\cos \left(\frac{\pi}{2} k_{1} \alpha_{1}\right)=0 \\
\cos \left(\frac{\pi}{2} k_{2} \alpha_{1}\right)=0
\end{array} .\right.
$$

This system admits only one solution, which means that we can delete only one frequency component. Figure 2 shows that the PSD of $\operatorname{TOC}\left(1,1, \alpha_{1}=1 / 3\right)$ presents a similar spectral density to that of $\operatorname{BOC}(1,1)$ but introduces additional zeros at $3 f_{s}, 9 f_{s}, 15 f_{s}, 21 f_{s} \ldots$

\section{Case 2: $M=2$ and $0<a_{1}<a_{2}<1$}

This case permits determination and elimination of the terms of both frequencies $k_{1} f_{s}$ and $k_{2} f_{s}$ by solving the following system of equations:

$$
\left\{\begin{array}{l}
\sum_{l=1}^{2}(-1)^{1+1} \cos \left(\frac{\pi}{2} k_{1} \alpha_{1}\right)=0 \\
\sum_{l=1}^{2}(-1)^{1+1} \cos \left(\frac{\pi}{2} k_{2} \alpha_{1}\right)=0
\end{array} .\right.
$$

The solution shows that the exact values of $\alpha_{1}$ and $\alpha_{2}$ are obtained when $k_{1}=3$ and $k_{2}=7$.

Figure 3 shows the PSD of both $\operatorname{BOC}(1,1)$ and BOC-AW $\left(1,1, \alpha^{(2)}\right)$ modulations with $\alpha_{1}=7.62 / 20$ and $\alpha_{2}=19.05 / 20$. Note that the PSD of BOC-AW $\left(1,1, \alpha^{(2)}\right)$

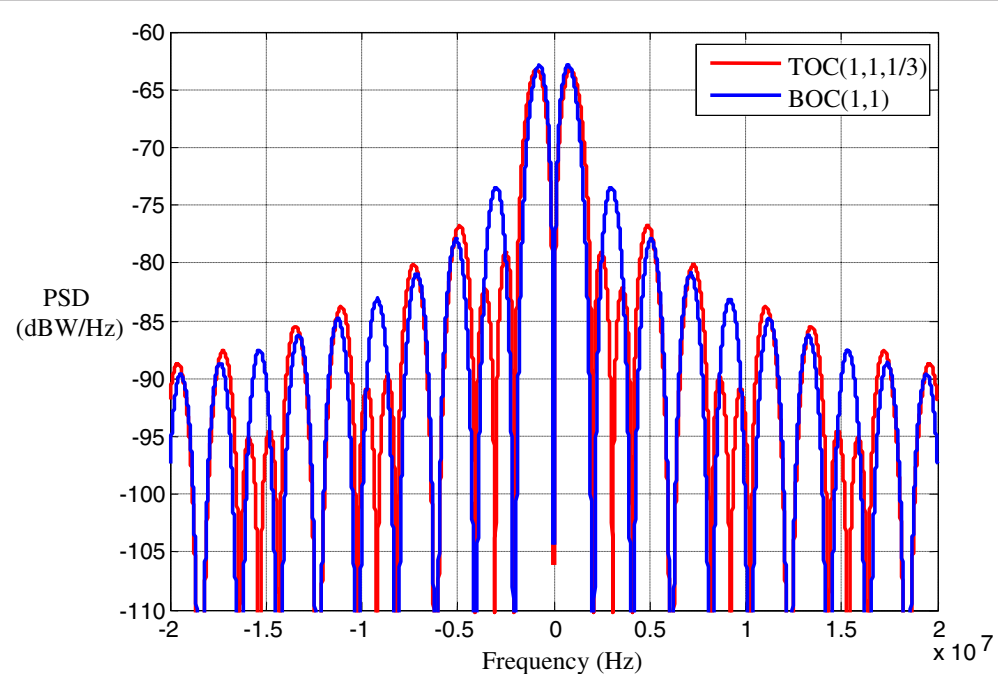

Figure 2 Normalized PSDs of TOC $\left(1,1, a_{1}=1 / 3\right)$ and BOC $(1,1)$. 


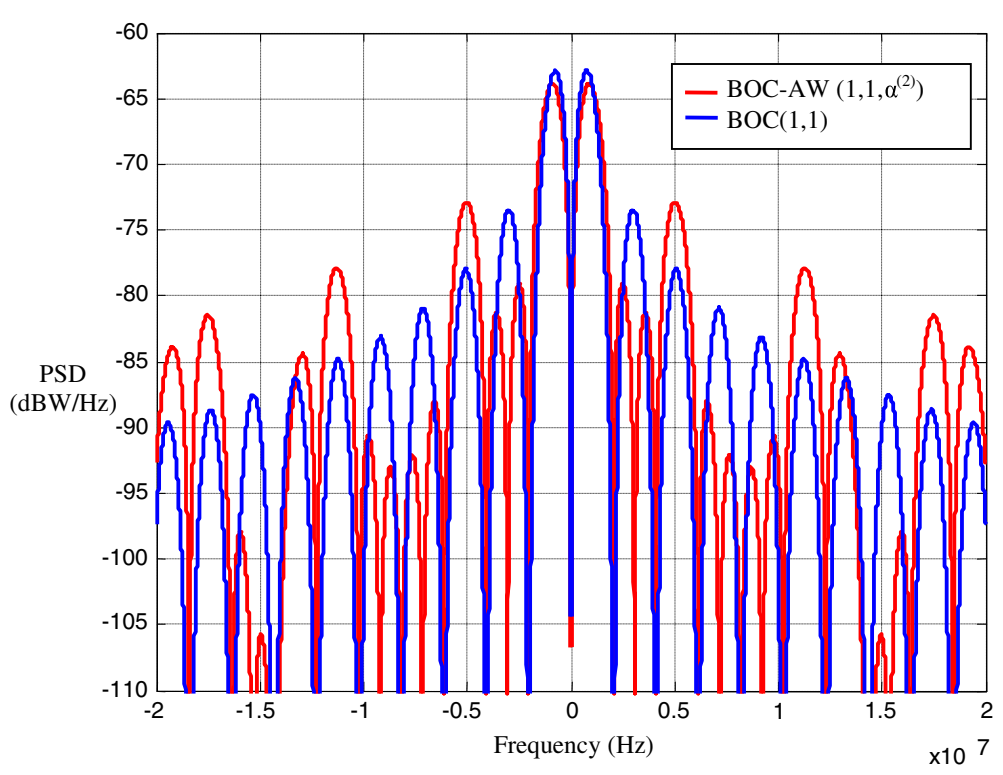

Figure 3 Normalized PSDs of BOC-AW $\left(1,1, a^{(2)}\right)$ and $\mathrm{BOC}(1,1)$.

introduces additional zeros at $3 f_{s}$ and $7 f_{s}$ and strengthens the other components of the PSD.

Case 3: $M=4$ and $0<a_{1}<a_{2}<a_{3}<a_{4}=1$

This case corresponds to $\operatorname{BOC}-\mathrm{AW}\left(1,1, \alpha^{(4)}\right)$ WF (Figure 1d). This WF is defined by four factors, $\alpha_{1}, \alpha_{2}$, $\alpha_{3}$, and $\alpha_{4}=1$, whose judicious choice eliminates the frequency components $3 f_{s}, 5 f_{s}$, and $7 f_{s}$ in the $\operatorname{BOC}(p, p)$ PSD.

In order to delete three frequency components that correspond to $k_{1}, k_{2}$, and $k_{3}$, the three values $\alpha_{1}, \alpha_{2}$, and $\alpha_{3}$ must be found by solving the following system of equations:

$$
\left\{\begin{array}{l}
\sum_{l=1}^{4}(-1)^{l+1} \cos \left(\frac{\pi}{2} k_{1} \alpha_{l}\right)=0 \\
\sum_{l=1}^{4}(-1)^{l+1} \cos \left(\frac{\pi}{2} k_{2} \alpha_{l}\right)=0 . \\
\sum_{l=1}^{4}(-1)^{l+1} \cos \left(\frac{\pi}{2} k_{3} \alpha_{l}\right)=0
\end{array}\right.
$$

Figure 4 shows the $\mathrm{BOC}(1,1)$ and the resulting $\mathrm{BOC}$ AW $\left(1,1, \alpha^{(4)}\right)$ PSDs. Note that the PSD of BOC-AW

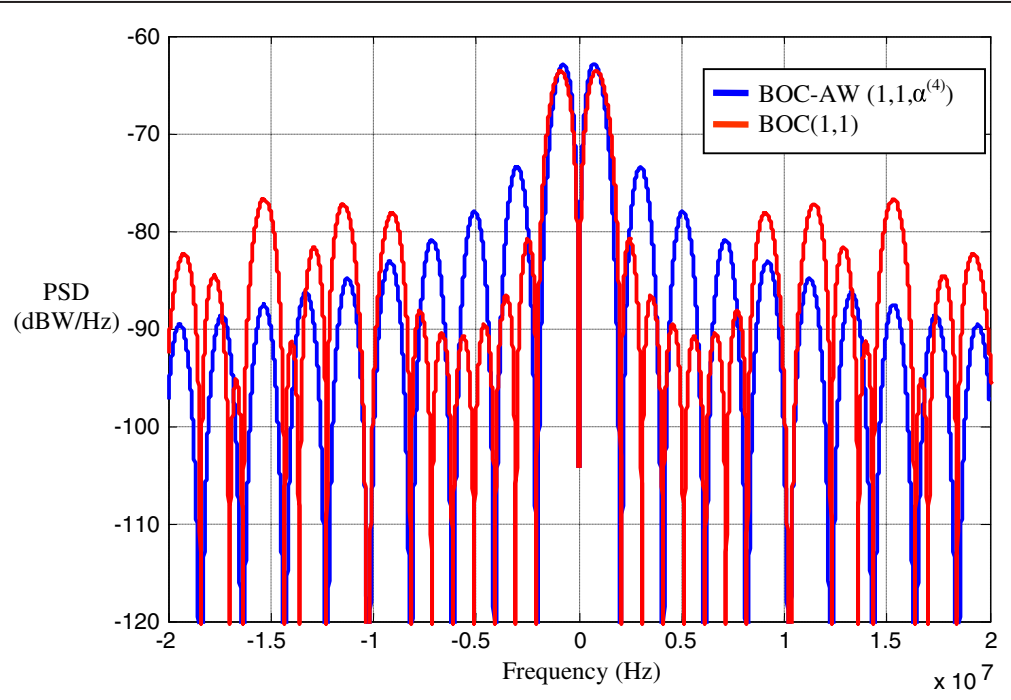

Figure 4 Normalized PSDs of BOC-AW $\left(1,1, a^{(4)}\right)$ and $\mathrm{BOC}(1,1)$. 
$\left(1,1, \alpha^{(4)}\right)$ compared to that of $\mathrm{BOC}(1,1)$ introduces additional zeros at $3 f_{s}, 5 f_{s}$, and $7 f_{s}$ and increases power at higher frequencies.

Case 4: $M=6$ and $0<a_{1}<a_{2}<a_{3}<a_{4}<a_{5}<a_{6}=1$

This last case represents the BOC-AW $\left(1,1, \alpha^{(6)}\right)$ WF (Figure 1e) with six factors, $\alpha^{(6)}$. BOC-AW $\left(1,1, \alpha^{(6)}\right)$ is used with a judicious choice of factors, $\alpha^{(6)}$, to delete five frequency components $\left(3 f_{s}, 5 f_{s}, 7 f_{s}, 9 f_{s}\right.$, and $\left.11 f_{s}\right)$ in BOC $(1,1)$ PSD.

To do this, we must solve the system of equations given as

$$
\left\{\sum_{l=1}^{6}(-1)^{1+1} \cos \left(\frac{\pi}{2} k \alpha_{l}\right)=0\right.
$$

for $k=k_{1}, \ldots, \mathrm{k}_{5}$.

Figure 5 shows the $\operatorname{BOC}(1,1)$ and the resulting $\mathrm{BOC}$ AW $\left(1,1, \alpha^{(6)}\right)$ PSDs. Note that the PSD of BOC-AW $\left(1,1, \alpha^{(6)}\right)$ compared to that of $\mathrm{BOC}(1,1)$ introduces additional zeros at $3 f_{s}, 5 f_{s}, 7 f_{s}, 9 f_{s}$, and $11 f_{s}$ and increases power at higher frequencies. Figure 6 shows the ACFs of different BOC-AW versions and BOC modulations.

It is clear that the ACFs of BOC-AW yield sharper peaks with respect to that of $\operatorname{BOC}(1,1)$. This causes an improvement of the code tracking performance, as we are going to see in the last part of this paper.

\section{Noise-induced code tracking error}

The Cramér-Rao lower bound (CRLB) is the lower bound of the root mean square error (RMSE) for any estimate of a nonrandom parameter. The CRLB can be expressed as $[9,15]$

$$
\begin{aligned}
\text { CRLB } & =-\frac{B_{L}}{\left(C / N_{0}\right) R_{s s}^{\prime \prime}(0)} \\
& =\frac{B_{L}}{\left(C / N_{0}\right)(2 \pi)^{2} \int_{-\infty}^{\infty} f^{2} G_{s}(f) d f}
\end{aligned}
$$

where $B_{L}$ refers to the loop bandwidth of the code tracking loop, $C / N_{0}$ is the carrier-to-noise ratio, and $R_{s S}^{\prime \prime}(\tau)$ and $G_{s}(f)$ are respectively the ACF and the PSD of the signal. In order to understand how the code tracking noise behaves for the set of modulations considered in this paper, we present in Figures 7, 8, 9 the CRLB using respectively 5, 12 , and $24 \mathrm{MHz}$ receiving bandwidths (double-sided) and $B_{L}=0.2 \mathrm{~Hz}$.

Figure 7 shows that the proposed BOC-AW modulations, with $5 \mathrm{MHz}$ bandwidth, provide a much better code tracking accuracy than TMBOC $(6,1,4 / 33)$, CBOC $(6,1,1 / 11)$ pilot, $\operatorname{CBCS}([1,-1,1,-1,1,-1,1,-1,1,1], 1,20 \%)$, and $\operatorname{BOC}(1,1)$ modulations. As we can recognize in Figure 8, for $12 \mathrm{MHz}$ receiving bandwidth, the best performance is given by $\operatorname{CBCS}([1,-1,1,-1,1,-1,1,-1,1,1], 1,20 \%)$, followed by BOC-AW $\left(1,1, \alpha^{(2)}\right)$, whereas all other modulations clearly outperform BOC-AW $\left(1,1, \alpha^{(4)}\right)$ and BOC-AW $\left(1,1, \alpha^{(6)}\right)$.

Figure 9 shows that the proposed modulations BOCAW $\left(1,1, \alpha^{(2)}\right)$ and BOC-AW $\left(1,1, \alpha^{(4)}\right)$ with $24 \mathrm{MHz}$ bandwidth provide the best code tracking accuracy. However, all other modulations clearly outperform $\operatorname{BOC}-\mathrm{AW}\left(1,1, \alpha^{(6)}\right)$.

\section{RMS bandwidth and cumulative PSDs}

The root mean square bandwidth (RMSB) can also be seen as another way of interpreting the CRLB or as the

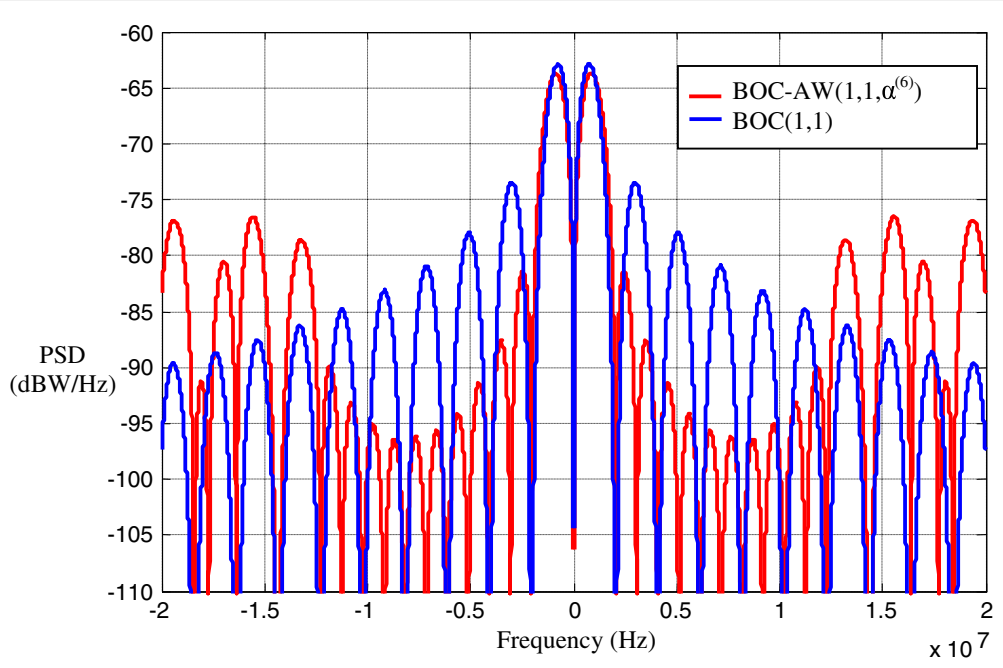

Figure 5 Normalized PSDs of BOC-AW $\left(1,1, a^{(6)}\right)$ and $\mathrm{BOC}(1,1)$. 


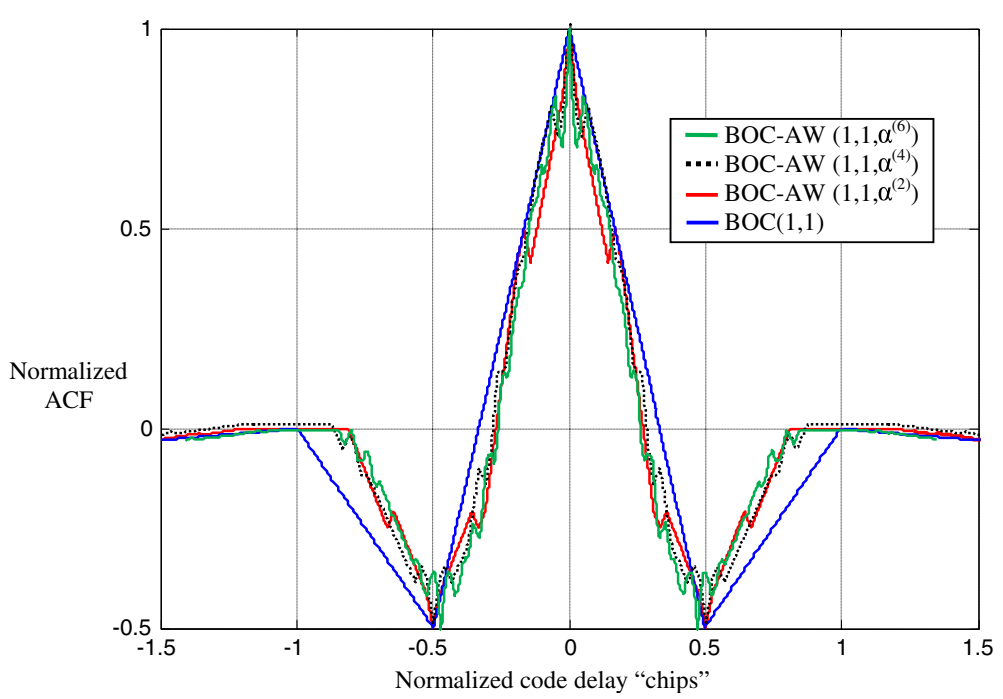

Figure 6 Normalized ACFs of BOC-AW $\left(1,1, a^{(2)}\right)$, BOC-AW $\left(1,1, a^{(4)}\right)$, BOC-AW $\left(1,1, a^{(6)}\right)$, and BOC $(1,1)$.

Gabor bandwidth of a signal [9]. The RMSB can be expressed as $[1,9]$

$$
\beta_{r m s}\left(B_{r}\right)=\sqrt{\int_{-B_{r} / 2}^{B_{r} / 2} f^{2} \overline{\mathrm{G}}_{s}(f) d f}
$$

where $\bar{G}_{s}(f)$ is the normalized PSD over receiver frontend bandwidth $B_{r}$.

Figures 10 and 11 show respectively the RMSB and the cumulative normalized PSDs of different modulations. As we can notice, for the $24 \mathrm{MHz}$ receiving bandwidth, the RMSBs of BOC-AW $\left(1,1, \alpha^{(2)}\right)$ and BOC-
$\operatorname{AW}\left(1,1, \alpha^{(4)}\right)$ are much greater than that of any other modulation, while the RMSB of BOC-AW $\left(2, \alpha^{(6)}\right)$ is the smallest. Ninety percent of the BOC-AW $\left(1,1, \alpha^{(2)}\right)$ power is reached within a frequency band less than 12 $\mathrm{MHz}$. This is approximately the same case for CBCS $([1,-1,1,-1,1,-1,1,-1,1,1], 1,20 \%)$ modulation. Nevertheless, $90 \%$ of both TMBOC $(6,1,4 / 33)$ and CBOC $(6,1,1 / 11)$ powers is situated in a frequency band greater than $12 \mathrm{MHz}$. The bandwidths including 90\% of the BOC-AW $\left(1,1, \alpha^{(4)}\right)$ and BOC-AW $\left(1,1, \alpha^{(6)}\right)$ powers are much wider than those including that of BOC

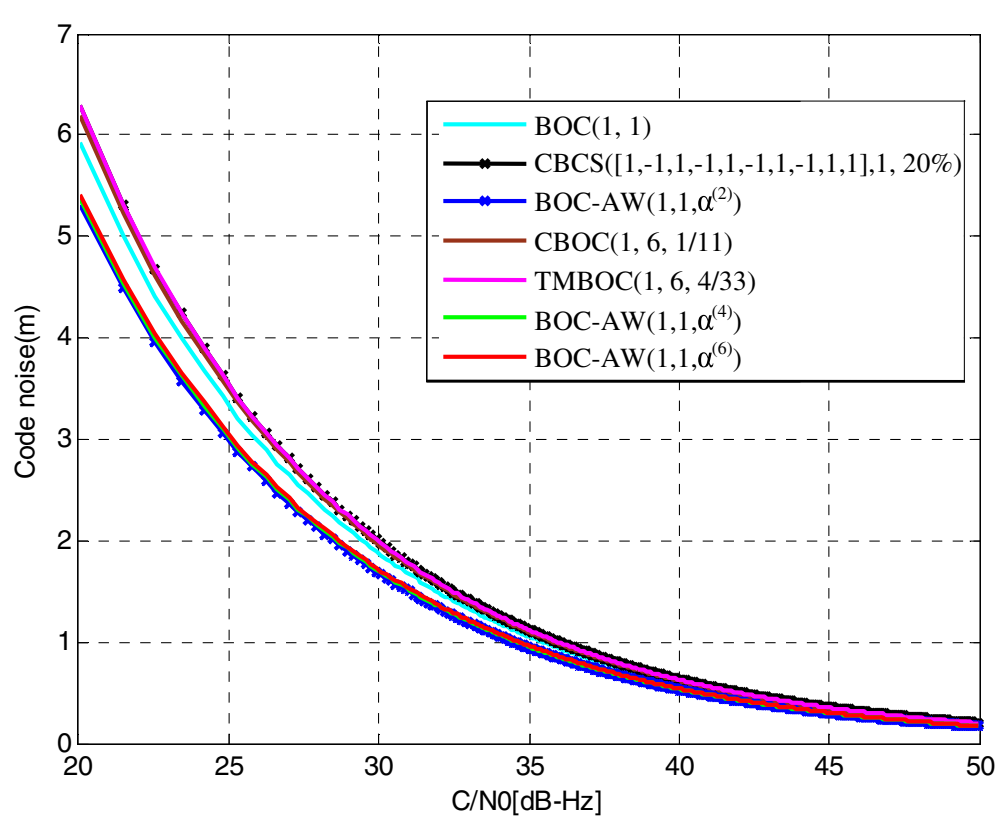

Figure 7 CRLB of TOC(1,1,1/3), BOC(1,1), CBCS([1,-1,1,-1,1,-1,1,-1,1,1],1,20\%), BOC-AW(1,1, $\left.a^{(2)}\right), \operatorname{CBOC}(6,1,1 / 11), \operatorname{TMBOC}(6,1,4 / 33)$, $\operatorname{BOC}-A W\left(1,1, a^{(4)}\right)$, and $B O C-A W\left(1,1, a^{(6)}\right)$ to $5 \mathrm{MHz}$ receiving bandwidth. 


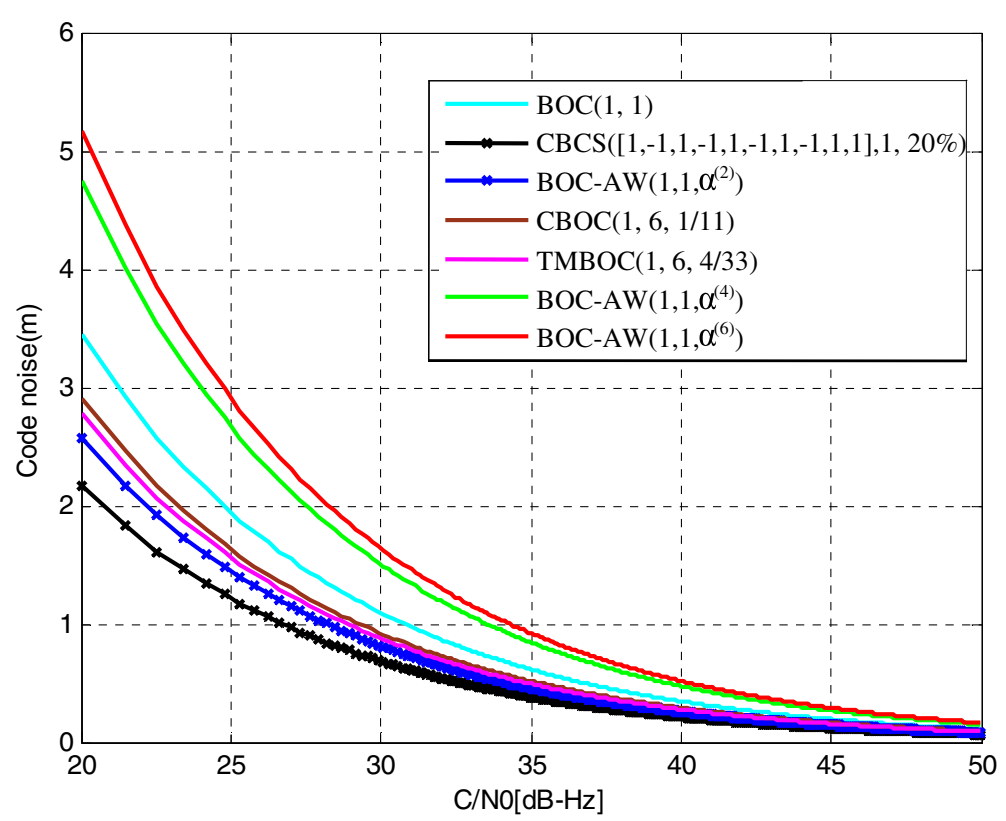

Figure 8 CRLB of TOC $(1,1,1 / 3)$, BOC(1,1), CBCS([1,-1,1,-1,1,-1,1,-1,1,1],1,20\%), BOC-AW(2, $\left.a^{(2)}\right), \operatorname{CBOC}(6,1,1 / 11)$, TMBOC(6,1,4/33), $\operatorname{BOC}-A W\left(1,1, a^{(4)}\right)$, and BOC-AW $\left(1,1, a^{(6)}\right)$ to $12 \mathrm{MHz}$ receiving bandwidth.

$(1,1), \quad \mathrm{CBCS}([1,-1,1,-1,1,-1,1,-1,1,1], 1,20 \%), \mathrm{TMBOC}$ $(6,1,4 / 33)$, and $\mathrm{CBOC}(6,1,1 / 11)$ powers.

Figure 12 shows the RMSBs of $\operatorname{BOC}(2,2), \operatorname{BOC}(2,1)$, and $\mathrm{BOC}-\mathrm{AW}\left(2,1, \alpha^{(2)}\right)$ modulations with $p=2$ and $q=1$. As we can recognize, for 5,12 , and $24 \mathrm{MHz}$ receiving bandwidths, the RMSB of BOC-AW $\left(2,1, \alpha^{(2)}\right)$ modulation is much greater than those of $\operatorname{BOC}(2,2)$ and $\operatorname{BOC}(2,1)$ modulations.

\section{Spectral separation coefficient}

SSC is a very important tool to design a new signal with better relevance for coexistence with GNSS signals in the same frequency band. In fact, the SSC concept provides a measure of the noise power output from a receiver when certain signals, with given spectra, are incident at its input. This shows that the fundamental measure is a cross PSD $[10,16]$.

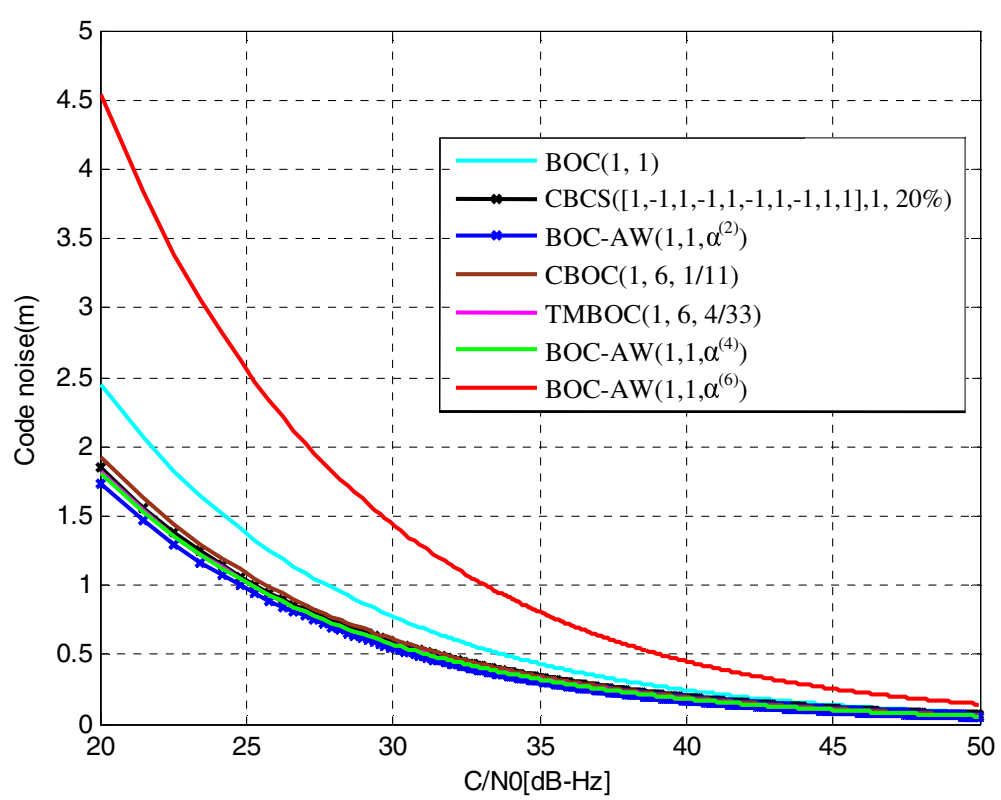

Figure 9 CRLB of TOC(1,1,1/3), BOC(1,1), CBCS([1,-1,1,-1,1,-1,1,-1,1,1],1,20\%), BOC-AW(1,1, $\left.a^{(2)}\right)$, CBOC(6,1,1/11), TMBOC(6,1,4/33), $\operatorname{BOC}-A W\left(1,1, a^{(4)}\right)$ and BOC-AW $\left(1,1, a^{(6)}\right)$ to $24 \mathrm{MHz}$ receiving bandwidth. 


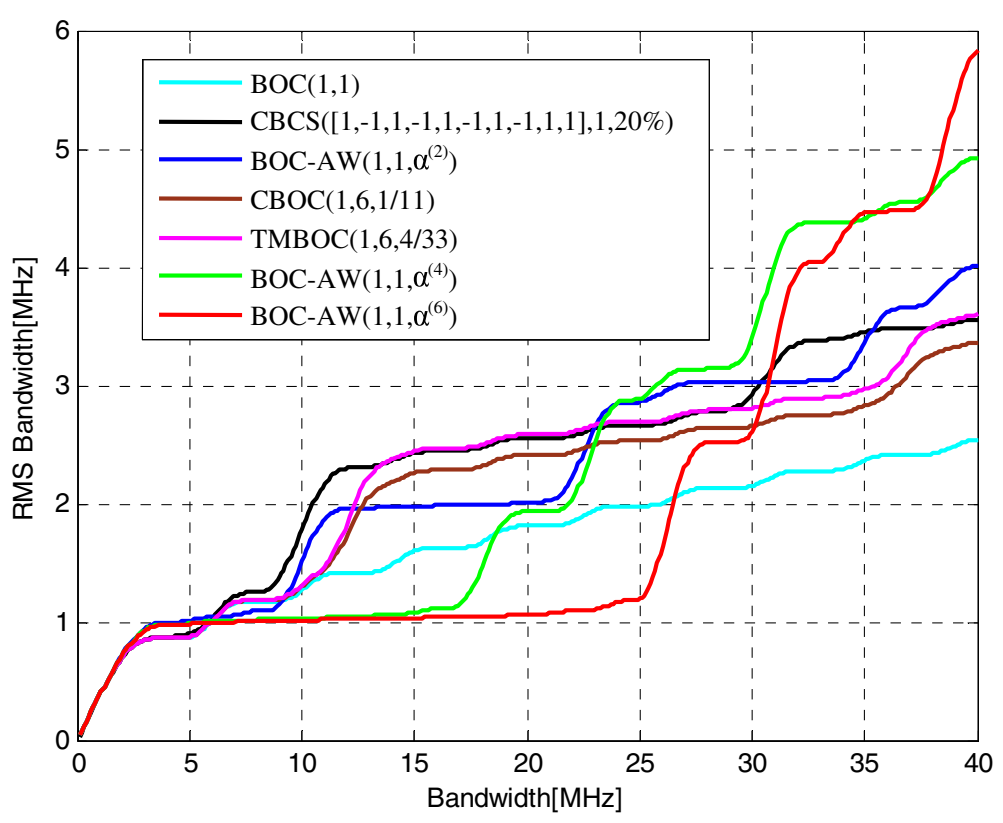

Figure 10 RMSEs of the different WFs.

The SSC between desired signal and interfering signal can be expressed as [1]

$$
k_{i s}=\int_{-B_{r} / 2}^{B_{r} / 2} G_{s}(f) G_{i}(f) d f
$$

where $B_{r}$ is the receiver front-end filter bandwidth, and $G_{s}(f)$ and $G_{i}(f)$ are respectively the normalized PSD of the desired signal and interfering signal. In Table 1, several SSC results are given for the case of infinite transmission bandwidth and a $24 \mathrm{MHz}$ receiver bandwidth.

As we can recognize from this table, the BOC-AW $\left(1,1, \alpha^{(6)}\right)$ WF presents better spectral separation with the GNSS WFs E1/L1. For example, the SSC for BOC-AW $\left(1,1, \alpha^{(6)}\right)$ with GPS coarse acquisition $(\mathrm{C} / \mathrm{A})$ code is $0.5 \mathrm{~dB}$ higher than that for $\operatorname{BOC}(1,1)$ with the same code. BOC-AW $\left(1,1, \alpha^{(2)}\right)$ and BOC-AW $\left(1,1, \alpha^{(4)}\right)$ WFs present

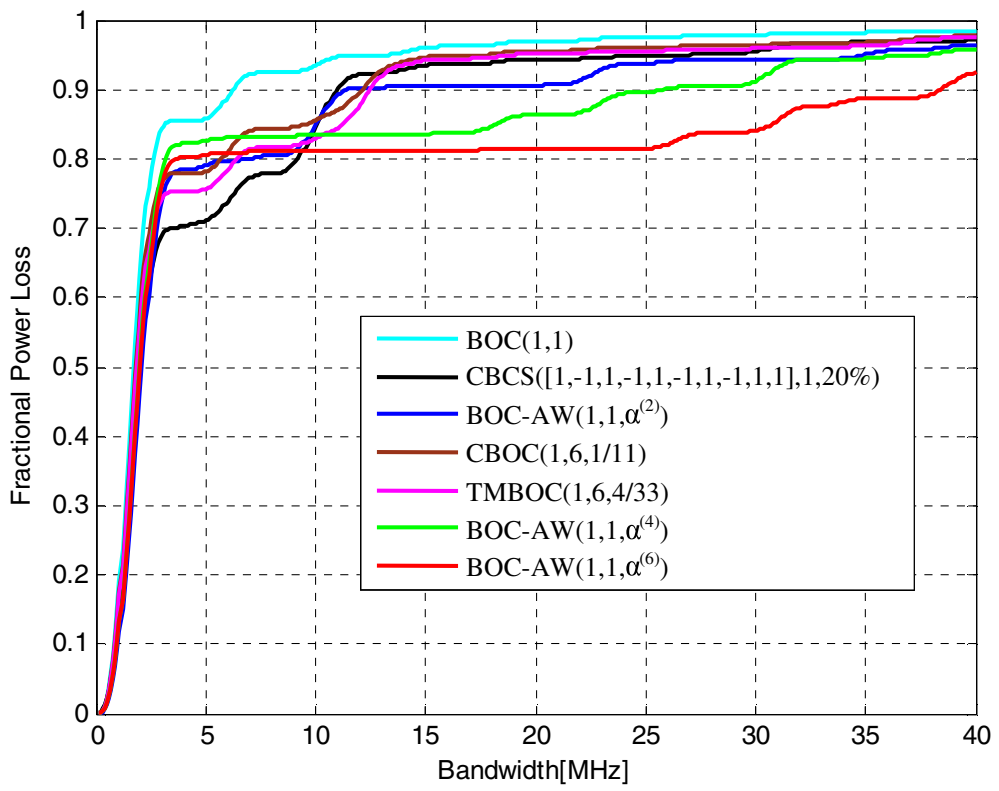

Figure 11 Cumulative normalized PSDs of different WFs. 


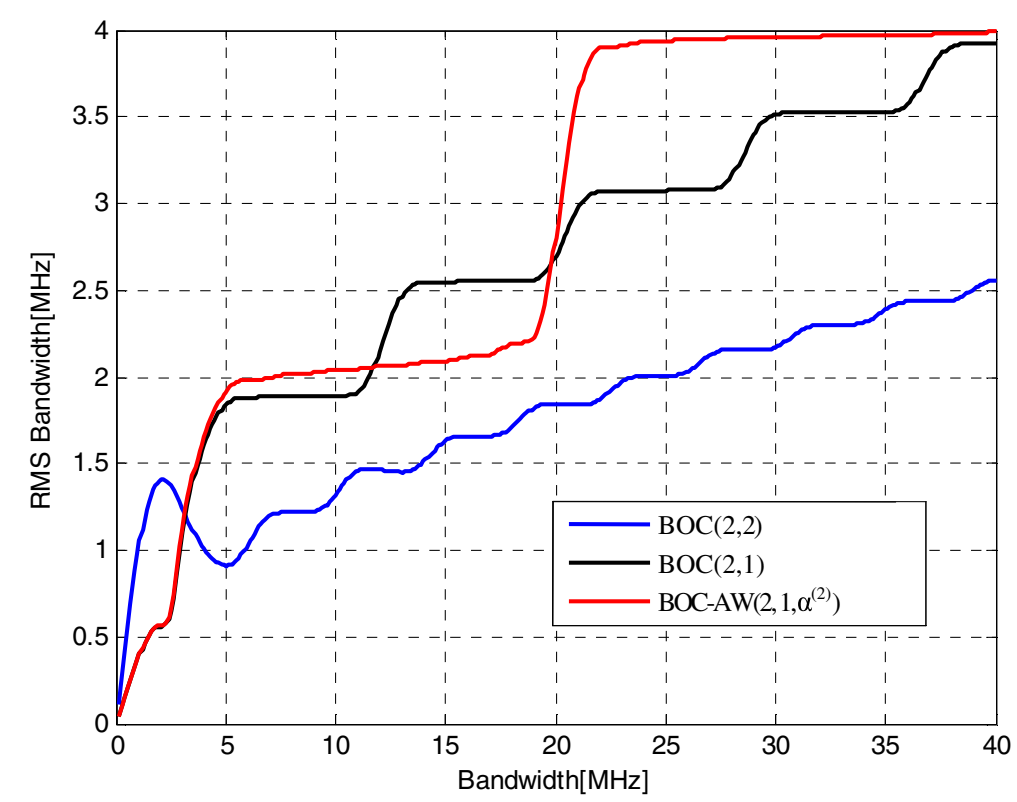

Figure 12 RMSEs of $\mathrm{BOC}(2,1), \mathrm{BOC}(2,2)$, and $\mathrm{BOC}-\mathrm{AW}\left(2,1, a^{(2)}\right)$.

less spectral overlapping with GPS P(Y), GPS C/A, GPS L1C, and Galileo E1 open service (OS) WFs. Also, the SSCs for BOC-AW $\left(1,1, \alpha^{(2)}\right)$ and BOC-AW $\left(1,1, \alpha^{(4)}\right)$ with GPS C/A code are respectively 0.48 and $0.27 \mathrm{~dB}$ higher than that for $\operatorname{BOC}(1,1)$ with the same code. However, the SSCs for BOC $(1,1)$ WF with GPS M code and with Galileo E1 public regulated service (PRS) are respectively higher than those for BOC-AW $\left(1,1, \alpha^{(2)}\right)$ and BOC-AW $\left(1,1, \alpha^{(4)}\right)$ with GPS M code and with Galileo E1 PRS.

\section{Real implementation of the proposed waveforms}

In reality, difficulty exists when directly applying the original WFs in the GNSS system. It is mainly due to the use of three-level WFs, including a zero level. In fact, this may lead to large power fluctuations of the radio frequency (RF) signal, which is a highly undesirable feature and represents a limitation in the practicality of our method. To overcome this problem, the proposed WFs were time multiplexed with $\operatorname{BOC}(M p, p)$ WFs. As a result, the zero transitions in BOC-AW WFs are occupied by a $\operatorname{BOC}(M p, p)$ WF. These optimized
BOC-AW (OBOC-AW) WFs are a constant envelope and bring another important quantity of energy at high frequencies that is added to that brought by the proposed original ones. This combination can be given as follows:

$$
S_{\mathrm{OBOC}-\mathrm{AW}}(t)=\left\{\begin{array}{ll}
S_{\mathrm{BOC}-\mathrm{AW}}(t) & \text { if }\left|S_{\mathrm{BOC}-\mathrm{AW}}(t)\right|=1 \\
S_{\mathrm{BOC}(M p, p)}(t) & \text { otherwise }
\end{array} .\right.
$$

OBOC-AW $\left(p, p, \alpha^{(2)}\right)$, OBOC-AW $\left(p, p, \alpha^{(4)}\right)$, and OBOC$\mathrm{AW}\left(p, p, \alpha^{(6)}\right)$ are shown respectively at the top, middle, and bottom of Figure 13.

The ACFs of both the BOC $(1,1)$ and OBOC-AW $\left(p, p, \alpha^{(M)}\right)$ WFs are illustrated in Figure 14. As illustrated in Figure 14, by performing this combination, a much sharper correlation peak can be achieved in practice. In addition, the resulting ACFs present side peaks with smaller levels compared to the $\mathrm{BOC}(1,1)$ ones. This will cause a small perturbation at the DLL in terms of ambiguity, and thus it will present the best performances in

Table 1 SSCs (dB) between BOC(1,1), TOC(1,1,1/3), BOC-AW, and GNSS E1/L1 signals

\begin{tabular}{llllll}
\hline Signal & GPS P (Y) & GPS C/A & GPS M & Galileo E1 PRS & GPS L1C \\
& $\begin{array}{l}\text { code } \\
\text { BPSK(10) }\end{array}$ & $\begin{array}{l}\text { code } \\
\text { BPSK(1) }\end{array}$ & $\begin{array}{l}\text { code } \\
\text { BOC(10,5) }\end{array}$ & BOCc(15,2.5) & $\begin{array}{l}\text { Galileo E1 OS } \\
\text { MBOC(6,1,1/11) }\end{array}$ \\
\hline BOC $(1,1)$ & -69.1191 & -73.5871 & -81.8083 & -103.3613 & -62.5287 \\
\hline TOC $(1,1,1 / 3)$ & -69.3055 & -73.8123 & -81.7546 & -103.1296 & -62.9200 \\
\hline BOC-AW $\left(1,1, a^{(2)}\right)$ & -69.5970 & -74.2102 & -81.7456 & -97.9512 & -63.3452 \\
\hline BOC-AW $\left(1,1, a^{(4)}\right)$ & -69.3835 & -73.7856 & -80.4395 & -97.3670 & -62.8448 \\
\hline BOC-AW $\left(1,1, a^{(6)}\right)$ & -69.6292 & -73.9962 & -84.1990 & -112.8249 & -63.0197 \\
\hline
\end{tabular}



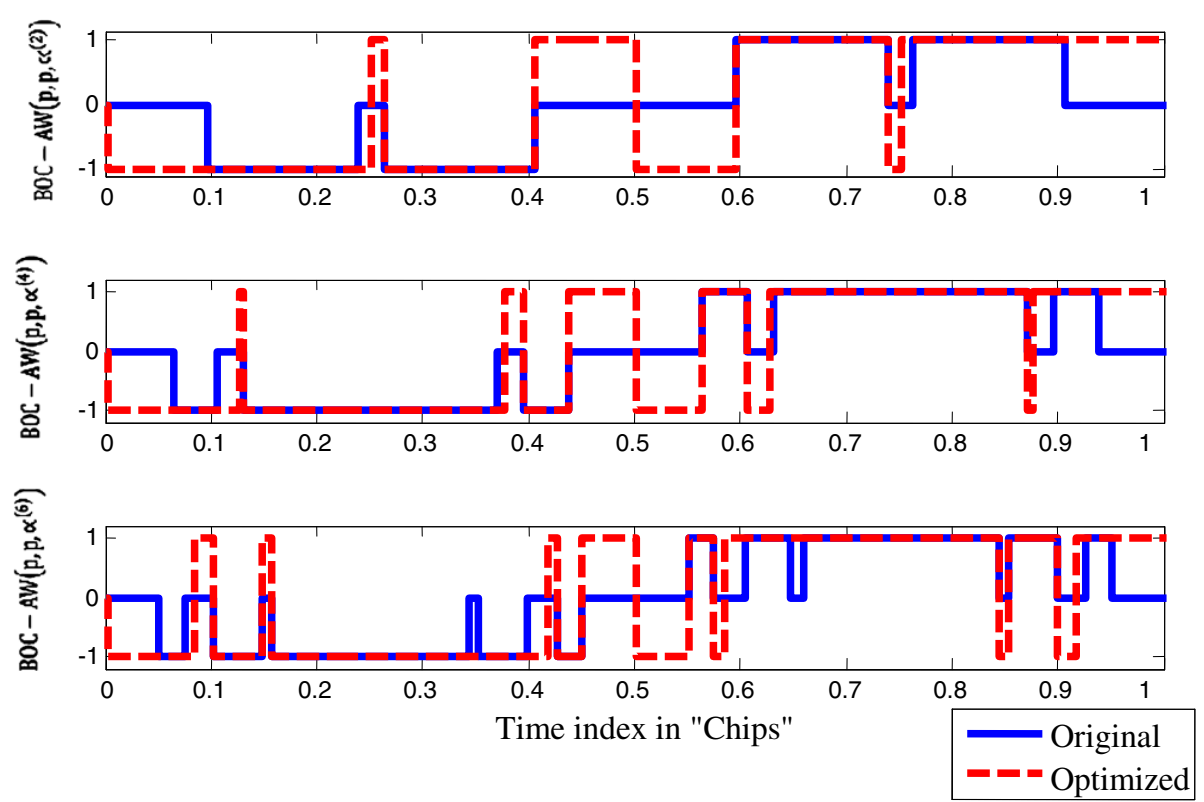

Figure 13 Original and optimized WFs.

positioning the receiver, as we are going to see in the last part of this paper.

\section{Simulation results}

Simulations were conducted to test the performances of the proposed WFs. For this reason, three situations were presented.

In the first situation, 11 schemes have been simulated. The first five were based respectively on $\mathrm{BOC}$ $(1,1)$, CBOC, TMBOC, TOC, and CBCS WFs. The last six schemes were based on our proposed WFs with BOC-AW configuration (BOC-AW $\left(1,1, \alpha^{(2)}\right)$, BOC-AW $\left(1,1, \alpha^{(4)}\right)$, and BOC-AW $\left.\left(1,1, \alpha^{(6)}\right)\right)$ and with OBOC-AW configuration (OBOC-AW $\left(1,1, \alpha^{(2)}\right)$, OBOC-AW $\left(1,1, \alpha^{(4)}\right)$, and OBOC-AW $\left.\left(1,1, \alpha^{(6)}\right)\right)$. In this first situation, we consider MP channel constructed with a line-of-sight (LOS) signal and a single reflected signal. Three different values of the pre-correlation bandwidth were chosen $(5,12$, and $24 \mathrm{MHz}$ ) to estimate the MP error envelopes of all 11 schemes. The MP signal has an amplitude of 0.5 and is varied in delay from 0 to $450 \mathrm{~m}$ with respect to the LOS delay. The MP error envelopes, which are calculated at the maximum points (when the MP signal is at $0^{\circ}$ 'in phase' or $180^{\circ}$ 'out of phase' with respect to the LOS) are used to calculate the running average errors. The principle of this consists in calculating the absolute envelope values and their cumulative sum with the aim of computing the average running errors. The norm used herein is that used in

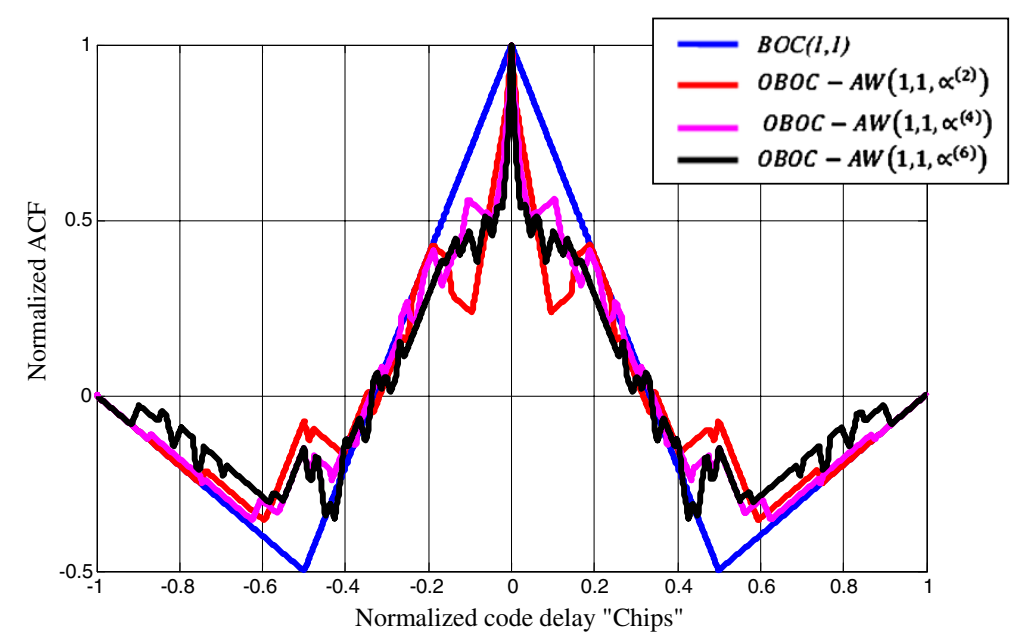

Figure 14 ACF of the optimized BOC-AW WFs. 


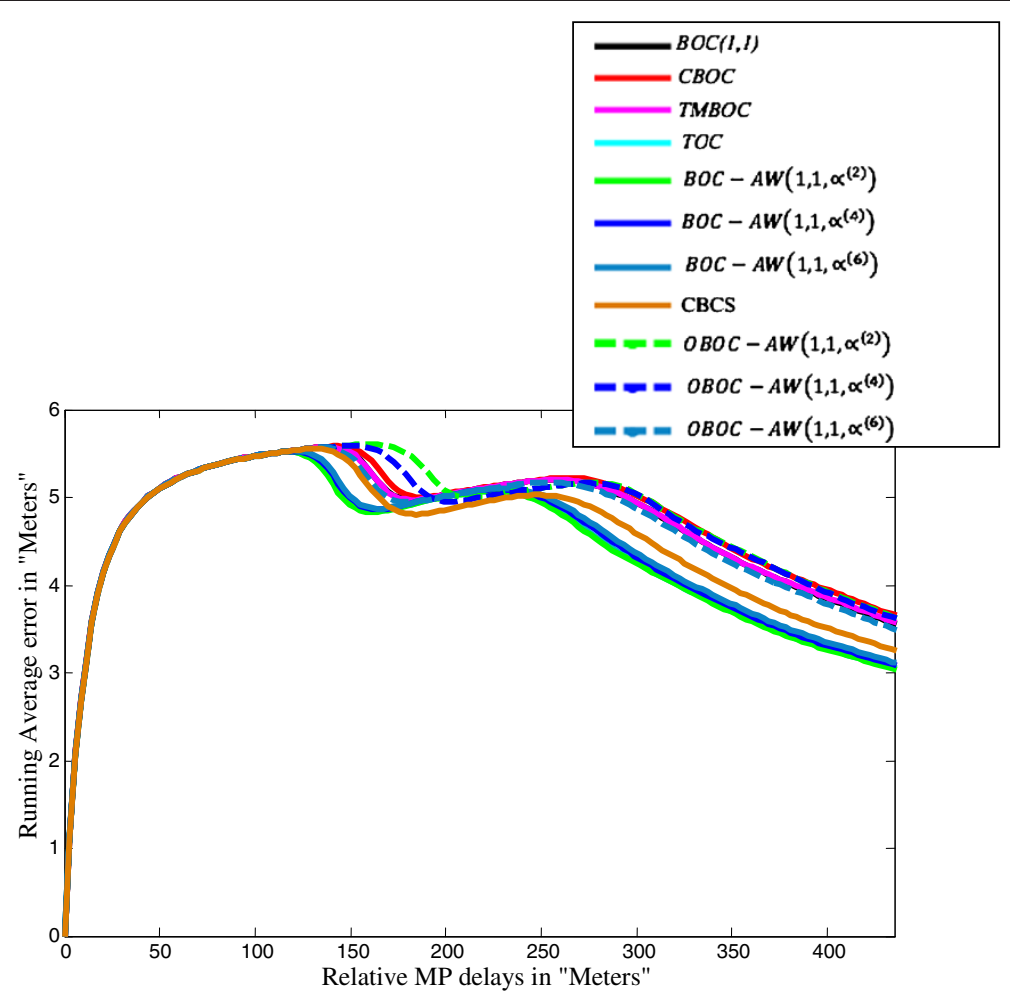

Figure 15 Running average errors of the different WFs with a pre-correlation bandwidth of $5 \mathrm{MHz}$.

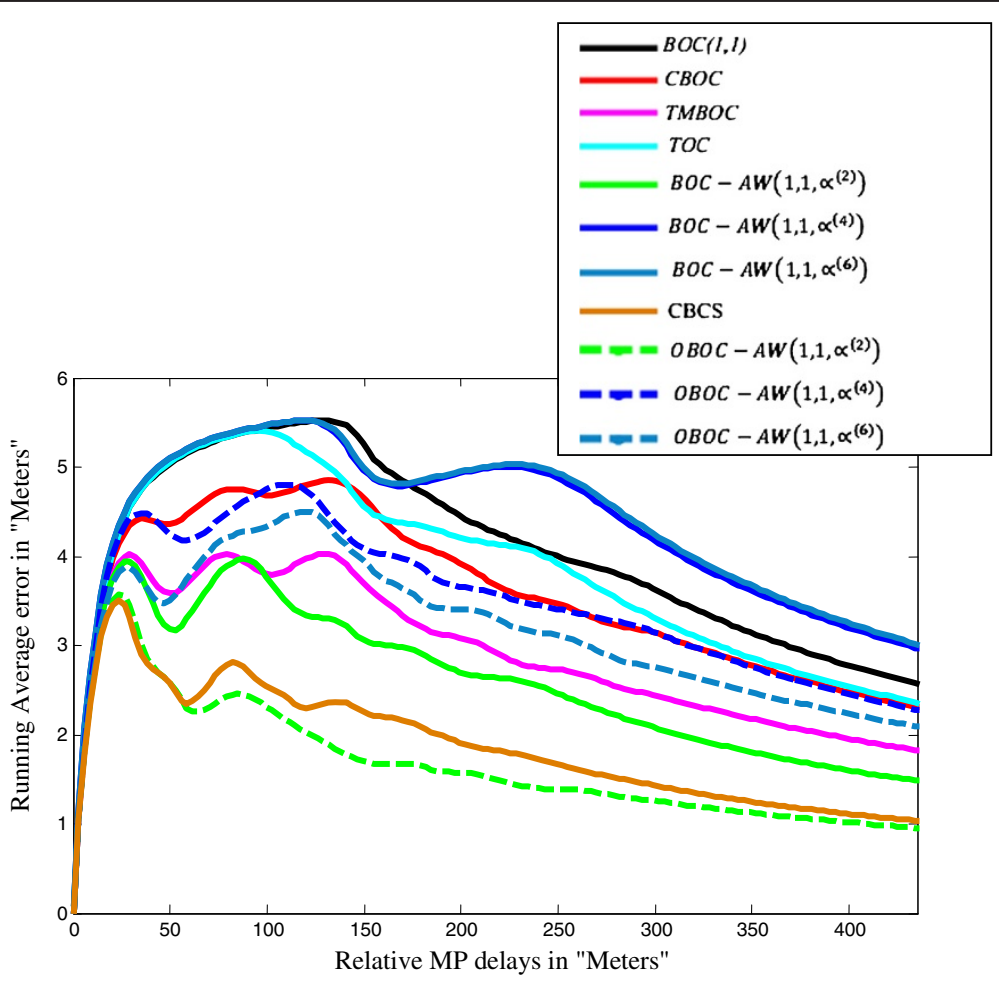

Figure 16 Running average errors of the different WFs with a pre-correlation bandwidth of $12 \mathrm{MHz}$. 


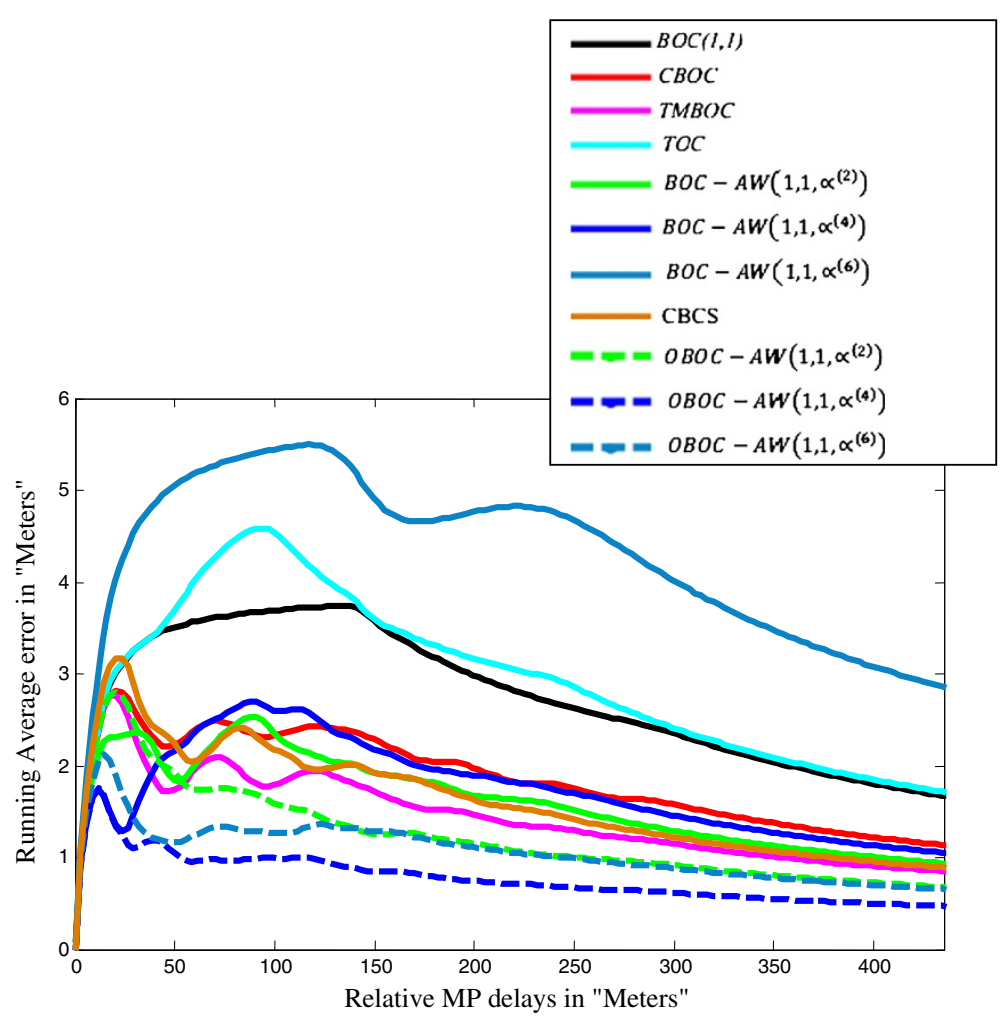

Figure 17 Running average errors of the different WFs with a pre-correlation bandwidth of $24 \mathrm{MHz}$.

reference [17]. The results, for the different band-limited ACFs, are shown in Figures 15, 16, 17.

As exposed in Figure 15, which corresponds to $5 \mathrm{MHz}$ pre-correlation bandwidth, the running average errors of the scheme based on our proposed WFs decrease toward small values from a delay which is greater than approximately $150 \mathrm{~m}$ with respect to the LOS. BOC-AW $\left(1,1, \alpha^{(2)}\right)$, BOC-AW $\left(1,1, \alpha^{(4)}\right)$, and BOC-AW $\left(1,1, \alpha^{(6)}\right)$ show the best performances for all MP delays greater than $125 \mathrm{~m}$ except for MP delays between 170 and $250 \mathrm{~m}$ where the best performances are given by the CBCS scheme. OBOC-AW $\left(1,1, \alpha^{(2)}\right)$, OBOC-AW $\left(1,1, \alpha^{(4)}\right)$, OBOC-AW $\left(1,1, \alpha^{(6)}\right)$, TOC, CBOC, BOC $(1,1)$, and TMBOC have similar performances for MP delays greater than approximately $200 \mathrm{~m}$ and present the worst schemes for MP delays in that range. However, for MP delays between 150 and $200 \mathrm{~m}$, it is the OBOC-AW $\left(1,1, \alpha^{(2)}\right)$ which represents the worst performances followed by OBOC-AW $\left(1,1, \alpha^{(4)}\right)$. This can be explained by the fact that the DSPs of our proposed BOCAW WFs present the largest principal lobes. Also, the performance degradation of the OBOC-AW WFs is due to the fact that their enhanced frequency components lie outside the $5 \mathrm{MHz}$ pre-correlation bandwidth.

In Figure 16 where the pre-correlation bandwidth is chosen equal to $12 \mathrm{MHz}$, both BOC-AW $\left(1,1, \alpha^{(4)}\right)$ and BOC-AW $\left(1,1, \alpha^{(6)}\right)$ WFs present the worst performances while $\mathrm{BOC}-\mathrm{AW}\left(1,1, \alpha^{(2)}\right)$ performs better than all the modulation schemes except for OBOC-AW $\left(1,1, \alpha^{(2)}\right)$ and CBCS. OBOC-AW $\left(1,1, \alpha^{(2)}\right)$ and CBCS show almost the same performances for delay values below $60 \mathrm{~m}$, while OBOC-AW $\left(1,1, \alpha^{(2)}\right)$ performs better for delay values greater than $60 \mathrm{~m}$. Finally, it should be noted that OBOC-AW $\left(1,1, \alpha^{(4)}\right)$ and OBOC-AW $\left(1,1, \alpha^{(6)}\right)$ WF performances are between those of $\mathrm{CBOC}$ and TMBOC.

In Figure 17, which corresponds to a $24 \mathrm{MHz}$ precorrelation bandwidth, the OBOC-AW $\left(1,1, \alpha^{(4)}\right)$ WFs present the best performances for all the band of variations of the MP delay with a maximum error of approximately $1.8 \mathrm{~m}$. The latter is followed by OBOC-AW $\left(1,1, \alpha^{(6)}\right)$ which gives the best performances than all the remaining schemes for delays greater than approximately $30 \mathrm{~m}$. For delays greater than approximately $60 \mathrm{~m}$, OBOC-AW $\left(1,1, \alpha^{(2)}\right)$ shows better performances than all the remaining WFs. Besides, BOC-AW $\left(1,1, \alpha^{(2)}\right)$ and BOC$\mathrm{AW}\left(1,1, \alpha^{(4)}\right)$ performances are close to those of CBCS, TMBOC, and CBOC and better than those of TOC and BOC $(1,1)$ WFs. BOC-AW $\left(1,1, \alpha^{(6)}\right)$ presents the worst case.

In addition to the MP perturbation, another limitation exists, which is the presence of noise. To test the robustness of our proposed WFs vis-à-vis the noise, we present the second situation. For this, all the previous schemes are firstly simulated. Then a comparison is accomplished between their code tracking RMSEs for three different values of the pre-correlation bandwidth 


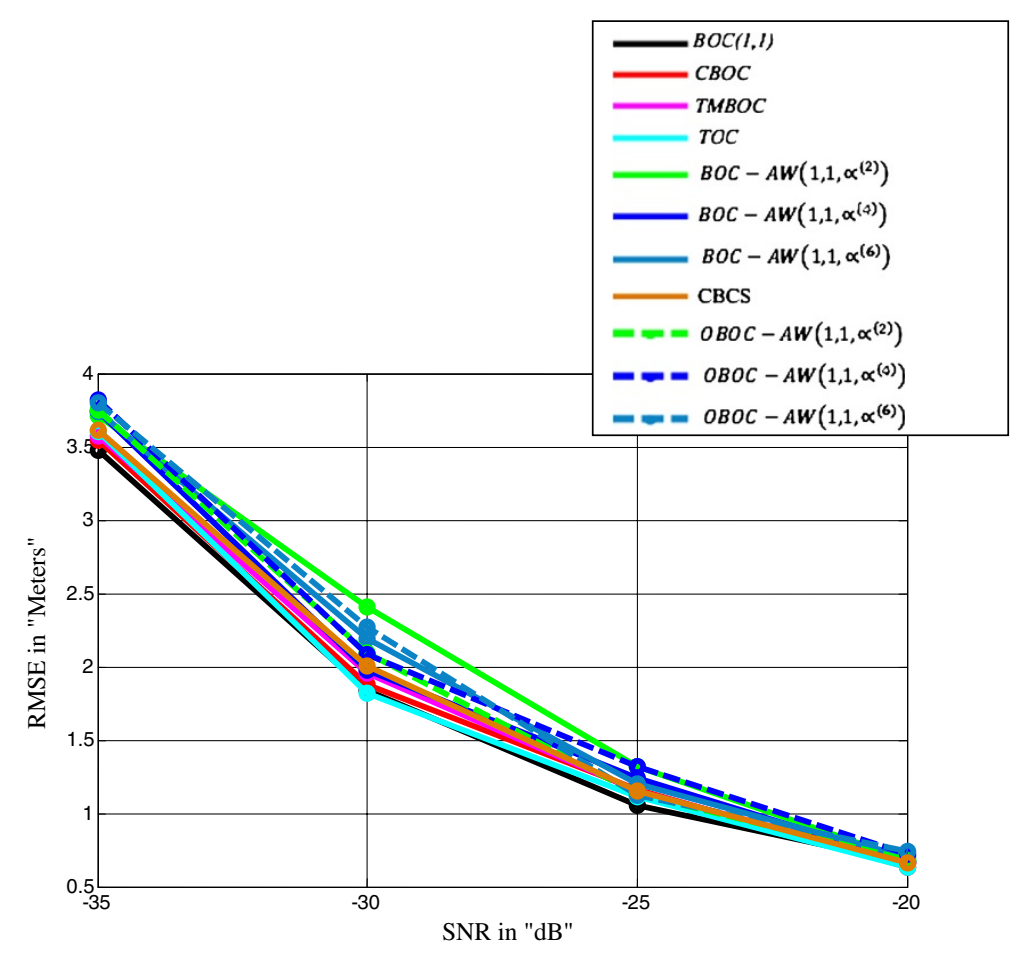

Figure 18 RMSEs of the different WFs with pre-correlation bandwidth of $5 \mathrm{MHz}$.

(5, 12, and $24 \mathrm{MHz})$. The results are shown respectively in Figures 18, 19, 20.

The RMSEs are represented versus signal-to-noise ratio (SNR) which varies from -35 to $-20 \mathrm{~dB}$. The SNR is defined as the $\left(C / N_{0}\right)$ divided by the RF signal bandwidth.
In Figure 18, corresponding to $5 \mathrm{MHz}$ pre-correlation bandwidth, the RMSEs of all our WFs approach those of all the other WFs. In Figure 19, we observe that for a 12 $\mathrm{MHz}$ pre-correlation bandwidth, the OBOC-AW $\left(1,1, \alpha^{(2)}\right)$ together with the CBCS WF shows the best performances regardless of the SNR value. For the $24 \mathrm{MHz}$

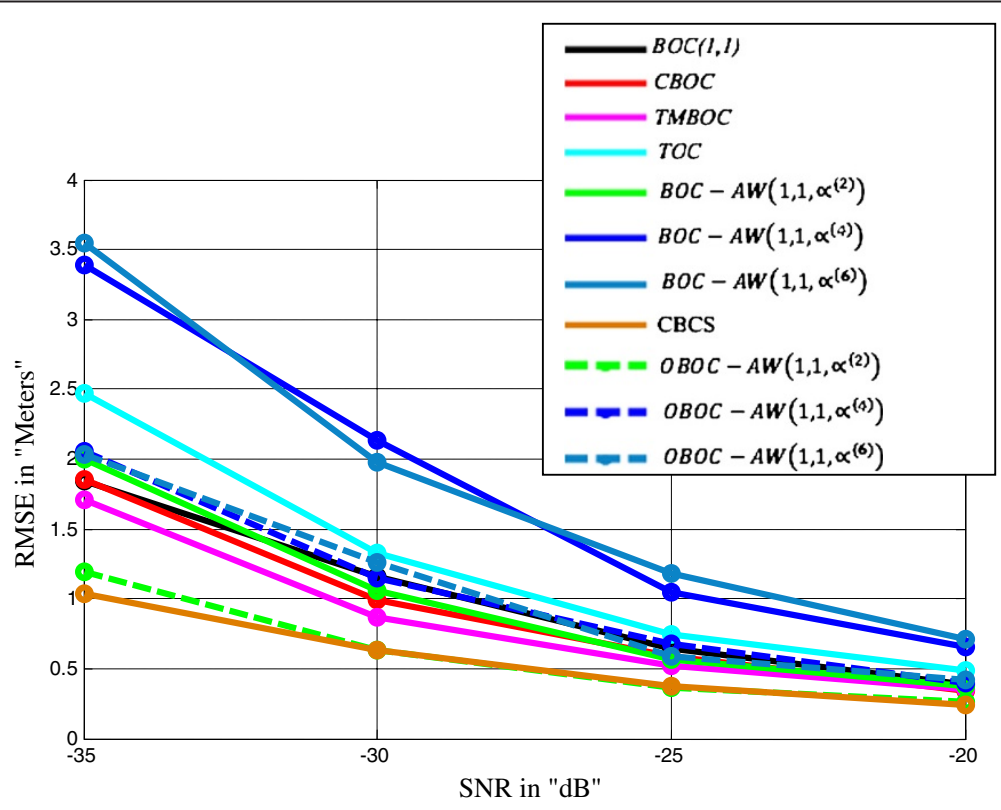

Figure 19 RMSEs of the different WFs with pre-correlation bandwidth of $12 \mathrm{MHz}$. 


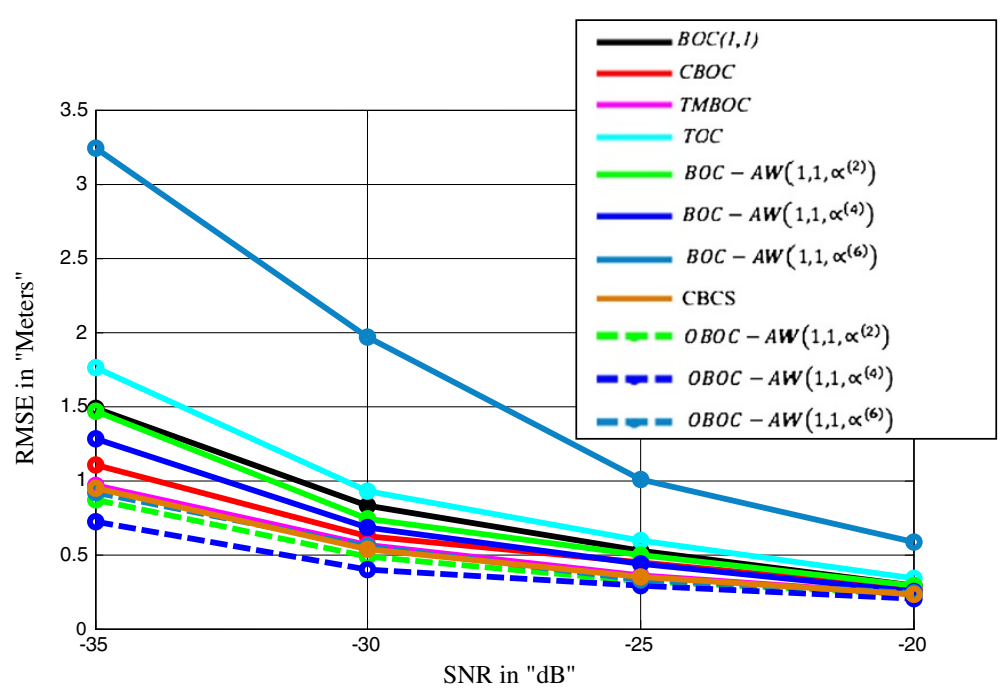

Figure 20 RMSEs of the different WFs with pre-correlation bandwidth of $24 \mathrm{MHz}$.

pre-correlation bandwidth, as shown in Figure 20, OBOC-AW $\left(1,1, \alpha^{(2)}\right)$ and OBOC-AW $\left(1,1, \alpha^{(4)}\right)$ present the smallest RMSEs in comparison with the other WFs, which shows their robustness concerning the noise and the efficiency of our proposed waveforms. OBOC-AW $\left(1,1, \alpha^{(4)}\right)$ presents the same RMSE with that of CBCS.

The final situation is realized to compare the RMSEs of our OBOC-AW WFs with those of BOC $(15,2.5)$ WF which has a high modulation order and lies in the same frequency band. The simulation results for 5, 12, and $24 \mathrm{MHz}$ are given respectively in Figures 21, 22 and 23. As shown in these figures, our WFs present the best performances $v i s-\grave{a}-v i s$ the noise. This result is valid for all pre-correlation bandwidth values.

\section{Conclusions}

In this paper, efficient WFs for MP mitigation and interference reduction in GNSS system are proposed. For this purpose, both BOC-AW and OBOC-AW WF configurations were presented and compared to the existing BOC WFs. BOC-AW WFs, although they present better performances than BOC ones, might suffer from power fluctuations caused by zero level. However, this problem is completely resolved by using OBOC-AW WFs. Besides, due to their ACF forms, OBOC-AW WFs are shown to have superior performance improvement in terms of MP error and delay variation band reduction. Among these, the OBOC-AW $\left(1,1, \alpha^{(2)}\right)$ WF is found to be undoubtedly the best. In addition, the proposed

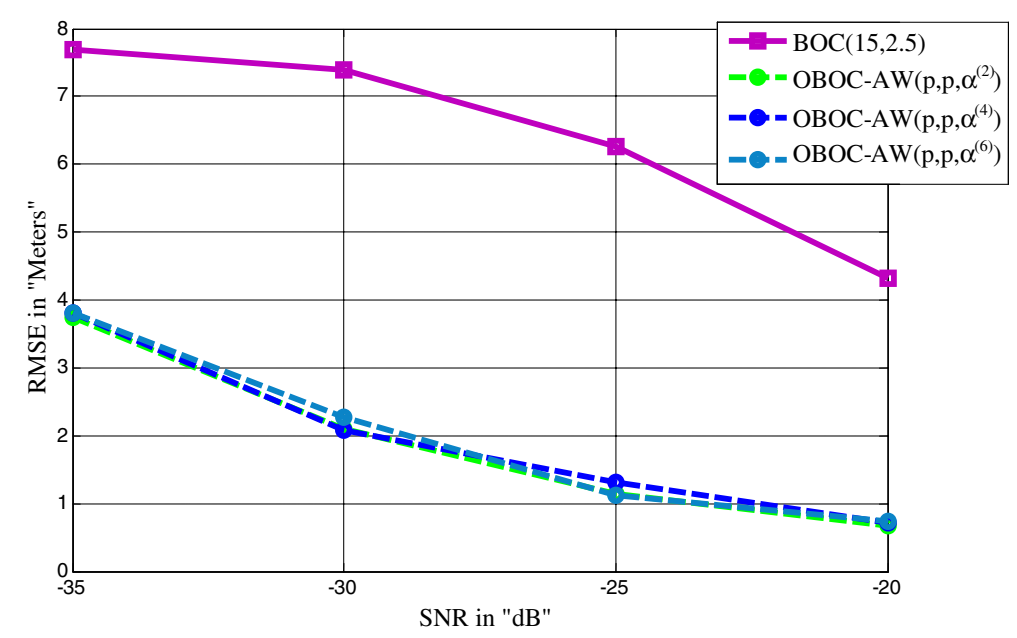

Figure 21 Comparison of RMSEs of our proposed WFs and $\mathrm{BOC}(15,2.5)$ with pre-correlation bandwidth of $5 \mathrm{MHz}$. 


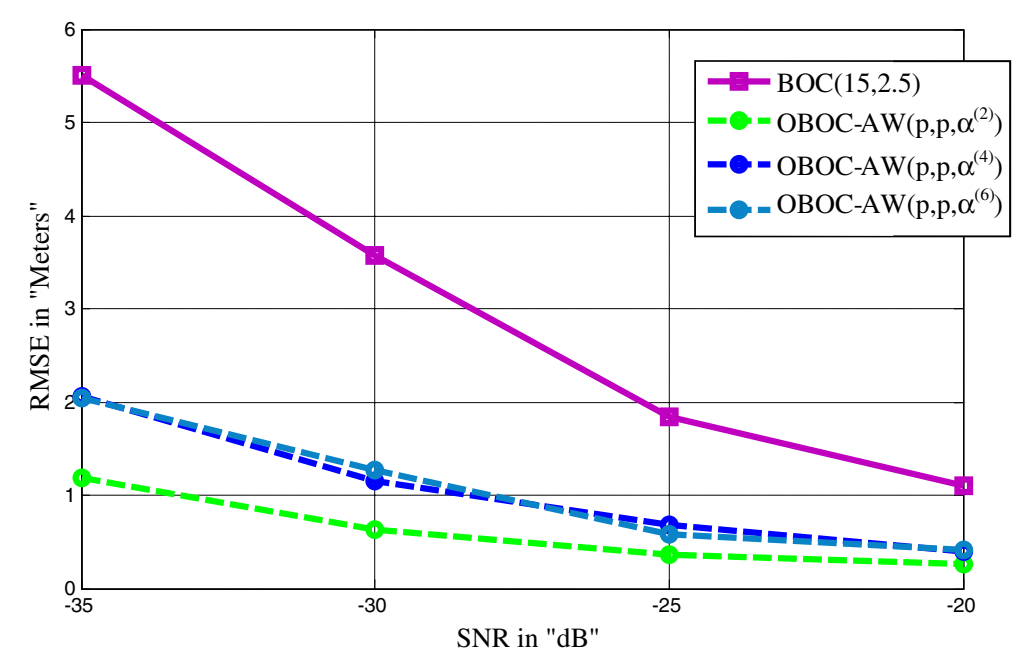

Figure 22 Comparison of RMSEs of our proposed WFs and $\mathrm{BOC}(15,2.5)$ with pre-correlation bandwidth of $12 \mathrm{MHz}$.

WFs present better resistance to noise and jamming due to their DSP distributions which present important quantities of power at high frequencies. Moreover, the scheme with these WFs works for both short/long and weak/strong MP.

\section{Appendix}

\section{Calculation of BOC-AW PSD}

Accepting the assumption given above, the PSD of the BOC-AW signal is given by

$$
G_{\left(p, q, \alpha^{(M)}\right)}(f)=\frac{\left|S_{\text {BOC-AW }\left(p, q, \alpha^{(M)}\right)}(f)\right|^{2}}{T_{c}}
$$

where $\left.S_{\mathrm{BOC}-\mathrm{AW}(p, q, \alpha}{ }^{(M)}\right)(f)$ is the Fourier transform of subcarrier WF $\left.s_{\mathrm{BOC}-\mathrm{AW}(p, q, \alpha}^{(M)}\right)(t)$.
In Equation 3, the Fourier transform of $\sum_{i=0}^{1} p_{l, \mathrm{i}}\left(t-m T_{s}\right)$ is given as

$$
\frac{\mathrm{e}^{-\mathrm{j} 2 \pi f m \mathrm{~T}_{\mathrm{s}}}}{\pi f \mathrm{e}^{\mathrm{j} f \mathrm{~T}_{\mathrm{s}}}}\left[\sin \left(\pi \mathrm{f}\left(1-\alpha_{2 l-1}\right) \mathrm{T}_{\mathrm{s}}\right)-\sin \left(\pi \mathrm{f}\left(1-\alpha_{2 l}\right) \mathrm{T}_{\mathrm{s}}\right)\right]
$$

Thus, the Fourier transform of the spreading symbol $s_{\mathrm{BOC}-\mathrm{AW}(p, q, \alpha)}(\mathrm{M})(t)$ is given as follows:

$$
\begin{aligned}
S_{\mathrm{BOC}-\mathrm{AW}\left(p, q, \alpha^{(M)}\right)}(f)= & \frac{1}{\pi f e^{j \pi f T_{s}}} \sum_{l=1}^{M / 2}\left[\sin \left(\pi f\left(1-\alpha_{2 l-1}\right) T_{s}\right)+\right. \\
& \left.-\sin \left(\pi f\left(1-\alpha_{2 l}\right) T_{s}\right)\right] \sum_{m=0}^{n-1}(-1)^{m} e^{-j 2 \pi f m T_{s}} .
\end{aligned}
$$

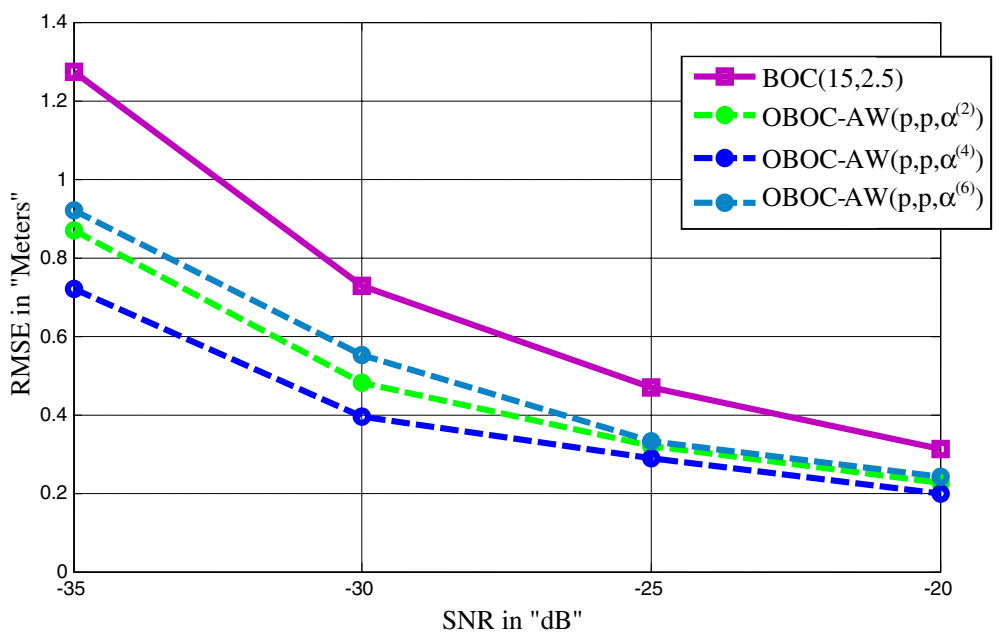

Figure 23 Comparison of RMSEs of our proposed WFs and $\mathrm{BOC}(15,2.5)$ with pre-correlation bandwidth of $24 \mathrm{MHz}$. 
The summation $\sum_{\mathrm{m}=0}^{\mathrm{n}-1}(-1)^{\mathrm{m}} \mathrm{e}^{-\mathrm{j} 2 \pi f \mathrm{~m} \mathrm{~T}_{\mathrm{s}}}$ in Equation 25 is given by [1]

$$
\sum_{m=0}^{n-1}(-1)^{m} e^{-j 2 \pi f m T_{s}}=j e^{-j(n-1) \pi f T_{s}}\left[\frac{\sin \left(n \pi f T_{s}\right)}{\cos \left(\pi f T_{s}\right)}\right]
$$

for $n$ even.

Substitution of Equation 26 into Equation 25 yields

$$
\begin{array}{r}
S_{\mathrm{BOC}-\mathrm{AW}}\left(n, \alpha^{(M)}\right) \\
\sum_{l=1}^{M / 2}\left[\sin \left(\pi f\left(1-\alpha_{2 l-1}\right) T_{s}\right)-\sin \left(\pi f\left(1-\alpha_{2 l}\right) T_{s}\right)\right] \\
\pi f e^{j \pi f T_{s}}
\end{array}
$$

and

$$
\sum_{m=0}^{n-1}(-1)^{m} e^{-j 2 \pi f m T_{s}}=e^{-j(n-1) \pi f T_{s}}\left[\frac{\cos \left(n \pi f T_{s}\right)}{\cos \left(\pi f T_{s}\right)}\right]
$$

for $n$ odd.

Substitution of Equation 28 into Equation 25, yields

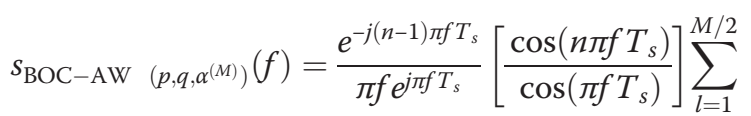

$$
\begin{aligned}
& \times\left[\sin \left(\pi f\left(1-\alpha_{2 l-1}\right) T_{s}\right)\right. \\
& \left.-\sin \left(\pi f\left(1-\alpha_{2 l}\right) T_{s}\right)\right] \text {. }
\end{aligned}
$$

Finally, the PSD for the BOC-AW signal is given as

$$
G_{(p, q, \alpha(M))}(f)=\frac{\left\{\sum_{l=1}^{M}(-1)^{1+1} \sin \left[\frac{\pi f}{2 f_{s}}\left(1-\alpha_{l}\right)\right]\right\}^{2} \sin ^{2}\left(\frac{\pi f}{f_{c}}\right)}{T_{c}\left(\alpha^{\prime}\right)(\pi f)^{2} \cos ^{2}\left(\frac{\pi f}{2 f_{s}}\right)}
$$

for $n$ even and

$$
G_{\left(p, q, \alpha^{(M)}\right)}(f)=\frac{\left\{\sum_{l=1}^{M}(-1)^{1+1} \sin \left[\frac{\pi f}{2 f_{s}}\left(1-\alpha_{l}\right)\right]\right\}^{2} \cos ^{2}\left(\frac{\pi f}{f_{c}}\right)}{T_{c}\left(\alpha^{\prime}\right)(\pi f)^{2} \cos ^{2}\left(\frac{\pi f}{2 f_{s}}\right)}
$$

for $n$ odd, where $\alpha^{\prime}$ indicates the active time where the signal adopts the values $\{-1,1\}$, and it is given as

$$
\alpha^{\prime}=\sum_{\mathrm{m}=1}^{\mathrm{M}}(-1)^{\mathrm{m}} \alpha_{\mathrm{m}}
$$

\section{Abbreviations}

ACF: autocorrelation function; BCS: binary coded symbol; BOC: binary offset carrier; BOC-AW: binary offset carrier with adjustable width; BPSK: binary phase shift keying; C/A: coarse acquisition; CBCS: composite binary coded symbols; CBOC: composite BOC; CRLB: Cramér-Rao lower bound; DLL: delay locked loop; GNSS: global navigation satellite systems; GPS: global positioning system; LOS: line of sight; MBOC: multiplexed BOC; MP: multipath; OBOC-AW: optimized BOC-AW; PRS: public regulated service; PSD: power spectral density; RMSEs: root mean square errors; SNR: signal-to-noise ratio; SSC: spectral separation coefficient; TOC: tertiary offset carrier; WF: waveform.

\section{Competing interests}

The authors declare that they have no competing interests.

\section{Acknowledgments}

The authors would like to thank Prof. Abderrahmane Bendaas, rector of the University of Bordj Bou Arréridj, for his moral support.

\section{Author details}

'LMSE Laboratory, Electronics Department, University of Bordj Bou Arréridj, Bordj Bou, Arréridj, Algeria. ${ }^{2}$ LIS Laboratory, Electronics Department, University of M'sila, M'sila, Algeria. ${ }^{3}$ DMMER Laboratory, Electronics Department, Ziane Achour University of Djelfa, Djelfa, Algeria.

Received: 16 May 2012 Accepted: 8 April 2013 Published: 9 May 2013

\section{References}

1. JW Betz, The offset carrier modulation for GPS modernization, in Proceedings of ION Technical Meeting (San Diego). 25-27 January 1999, pp. 639-648

2. JW Betz, Binary offset carrier modulations for radionavigation. J The Institute of Navigation 48, 227-246 (2001)

3. J Chen, YG Zhang, GB Liang, F Li, The anti-jamming performance of GNSS BOC signals in certain jamming environments, in Proceeding of ISAPE 2008: The 8th International Symposium on Antennas, Propagation and EM Theory, vol. 1-3 (Kunming). 2-5 November 2008

4. Galileo Open Service, Signal in space interface control document, European Space Agency/Galileo Joint Undertaking, Draft GAL OS SIS ICD/D.0. (2006)

5. GW Hein, JA Avila-Rodriguez, S Wallner, AR Pratt, MBOC: the new optimized spreading modulation recommended for Galileo L1 OS and GPS L1C, in Proceedings of the IEEE/ION Position, Location, and Navigation Symposium (San Diego), p. 25-27 April 2006, pp. 883-892

6. CJ Hegarty, JW Betz, A Saidi, Binary coded symbol modulations for GNSS, in Proceedings of the 60th Annual Meeting of the Institute of Navigation, IONNTM (Dayton). 7-9 June 2004

7. GW Hein, JA Avila-Rodriguez, L Ries, L Lestarquit, J-L Issler, J Godet, AR Pratt, Galileo Signal Task Force of the European Commission, A candidate for the Galileo L1 OS optimized signal, in Proceedings of the 18th International Technical Meeting of the Satellite Division of the Institute of Navigation (ION-GNSS) (Long Beach). 13-16 September 2005

8. Z Yao, M Lu, ZM Feng, Quadrature multiplexed BOC modulation for interoperable GNSS signals. Electron Lett 46, 1234-1236

9. JA Avila-Rodriguez, On generalized signal WFs for satellite navigation, PhD Thesis (University FAF Munich, Neubiberg, Germany, 2008)

10. AR Pratt, JIR Owen, BOC modulation WFs, in ION Proceedings, GPS 2003 Conference (Portland). 9-12 September 2003

11. AR Pratt, JIR Owen, Performance of GPS/Galileo receivers using m-PSK BOC signals, in Proceedings of the 2004 National Technical Meeting of the Institute of Navigation (San Diego). 26-28 January 2003

12. K Rouabah, D Chikouche, F Bouttout, R Harba, and P Ravier, "GPS/galileo multipath mitigation using the first side peak of double delta correlator", Wireless Personal Communications, 60, 2, pp. 321-333, (2010).

13. K Rouabah, D Chikouche, GPS/Galileo multipath detection and mitigation using closed-form solutions. Math Probl Eng, (2009). doi:10.1155/2009/ 106870

14. M Sahmoudi, MG Amin, Fast iterative maximum-likelihood algorithm (FIMLA) for multipath mitigation in the next generation of GNSS receivers. IEEE Trans Wirel Commun 7, 4362-4374 (2008)

15. J-A Avila-Rodriguez, T Pany, GW Hein, Bounds on signal performance regarding MP-estimating discriminators, in Proceedings of the International 
Technical Meeting of the Institute of Navigation, ION-GNSS 2006 (Fort Worth Convention Center, Fort Worth). 26-29 September 2006

16. JW Betz, Effect of partial-band interference on receiver estimation of $\mathrm{C} / \mathrm{NO}$ : theory, in Proceedings of the National Technical Meeting of the Institute of Navigation ION-NTM 2001 (Long Beach). 22-24 January 2001, pp. 16-27

17. M Irsigler, JA Avila-Rodriguez, GW Hein, Criteria for GNSS multipath performance assessment, in Proceedings of the International Technical Meeting of the Institute of Navigation (ION-GNSS 2005) (Long Beach).

13-16 September 2005

doi:10.1186/1687-1499-2013-124

Cite this article as: Flissi et al.: Performance of new BOC-AW-modulated signals for GNSS system. EURASIP Journal on Wireless Communications and Networking 2013 2013:124.

\section{Submit your manuscript to a SpringerOpen ${ }^{\circ}$} journal and benefit from:

- Convenient online submission

- Rigorous peer review

- Immediate publication on acceptance

- Open access: articles freely available online

- High visibility within the field

- Retaining the copyright to your article 